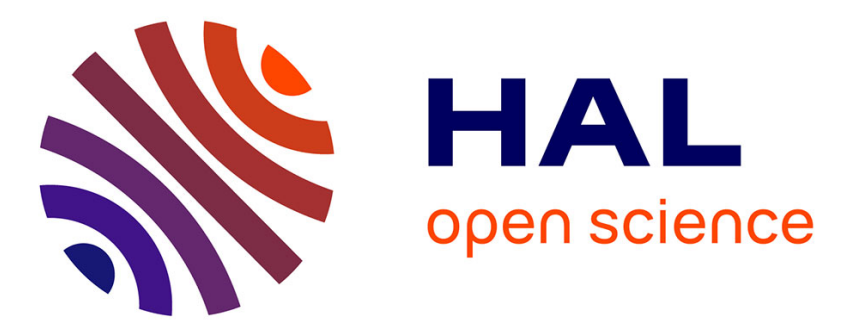

\title{
Carrières et extraction romaines dans le nord-est de la Gaule et en Rhénanie \\ Dagmar Lukas
}

\section{To cite this version:}

Dagmar Lukas. Carrières et extraction romaines dans le nord-est de la Gaule et en Rhénanie. Gallia - Archéologie de la France antique, 2002, Carrières antiques de la Gaule, 59, pp.155-174. 10.3406/galia.2002.3103 . hal-01912527

\section{HAL Id: hal-01912527 \\ https://hal.science/hal-01912527}

Submitted on 20 Jan 2020

HAL is a multi-disciplinary open access archive for the deposit and dissemination of scientific research documents, whether they are published or not. The documents may come from teaching and research institutions in France or abroad, or from public or private research centers.
L'archive ouverte pluridisciplinaire HAL, est destinée au dépôt et à la diffusion de documents scientifiques de niveau recherche, publiés ou non, émanant des établissements d'enseignement et de recherche français ou étrangers, des laboratoires publics ou privés.

\section{(ㅇ)(1) $\$$}

Distributed under a Creative Commons Attribution - NonCommercial - NoDerivatives| 4.0 


\title{
CARRIÈRES ET EXTRACTION ROMAINES DANS LE NORD-EST DE LA GAULE ET EN RHÉNANIE
}

\author{
Dagmar LUKAS
}

Mots-clés. Carrière, légion, inscription, graffiti, outil, technique d'extraction.

Key-words. Quarry, legion, inscription, graffiti, tool, quarrying technique.

Résumé. De nombreuses carrières de pierre de taille ont élé mises en exploitation dans les régions mosellane et rhénane aver l'arrivée des Romains. Les études archéologiques n'ont concerné jusqu'à présent qu'un petit nombre de sites d'extraction attribués à l'époque romaine, dont certains abritent des graffiti et des sculptures rupestres remarquables. Ils ont également livré un grand nombre de documents épigraphiques attestant l'intervention sur les lieux d'extraction de soldats détachés de leur unité et chargés de l'approvisionnement des chantiers militaires et civils dans les provinces frontalières du nord-est de l'Empire.

III'organisation des carrières était complexe el adaptée à la forte demande en matériau lithique. Les modes et techniques d'extraction ?ui sont lisibles grâce aux traces qu'ils ont laissées reflètent le savoir-faire et l'habileté des carriers, ainsi que les limites qui leur étaient inposées par le cadre naturel. Les Romains ont exploité les carrières à l'air libre comme en galeries souterraines en appliquant, selon Li contexte lithostratigraphique, une extraction parallèle ou perpendiculaire au lit naturel de la roche. Ies études consacrées aux carrières dans la région considérée sont pour la plupart anciennes, et de nombreuses questions restent ouvertes, qui ne pourront être traitées qu'avec

¿l'engagement de nouvelles recherches sur le terrain.

Abstract. With the arrival of the Romans, a great number of ashlar quarries were soon exploited in the Rhine and Moselle valleys. Until now, comparatively few Roman quarries have been examined, some of them having remarkable graffiti and rock carvings. Numerous epigraphic documents are showing how soldiers were detailed to work in the quarries supplying stone for military and civilian sites in the provinces of the northeast border.

The organization of the quarries was complex and adapted to the massive demand for stone material. The quarrying methods and techniques can be read from the marks they left and reflect the ability and knowledge of the quarrymen as well as the limits imposed by the physical context. The Romans exploited opencast as well as underground quarries and, depending on the lithostratigraphic context, cut trenches parallel or perpendicular to the bedrock. Most of the studies dealing with quarries in this region were carried on a long time. ago and many questions remain without answer. So new archaeological investigations need to be undertaken to examine over again these points

Dans le nord-est de la Gaule et en Rhénanie, la pierre ne joue pas de rôle déterminant dans l'architecture avant la romanisation. Le début de la conquête romaine peut donc être considéré comme une période charnière pour l'emploi de la pierre dans une région pourtant riche en ressources lithiques. Toutefois, l'immense couverture forestière et une longue tradition du travail du bois ont sans doute, dans un premier temps, freiné le succès de ce matériau, et il faut attendre le renforcement de la frontière du Rhin, consécutif à la défaite écrasante de Varus dans le saltus Teutoburgiensis en l'an 9 de notre ère, pour voir se développer l'industrie de la pierre dans la région. Des villes établies au bord du Rhin et de la Moselle, comme Mayence, Cologne et Trèves, connais- 


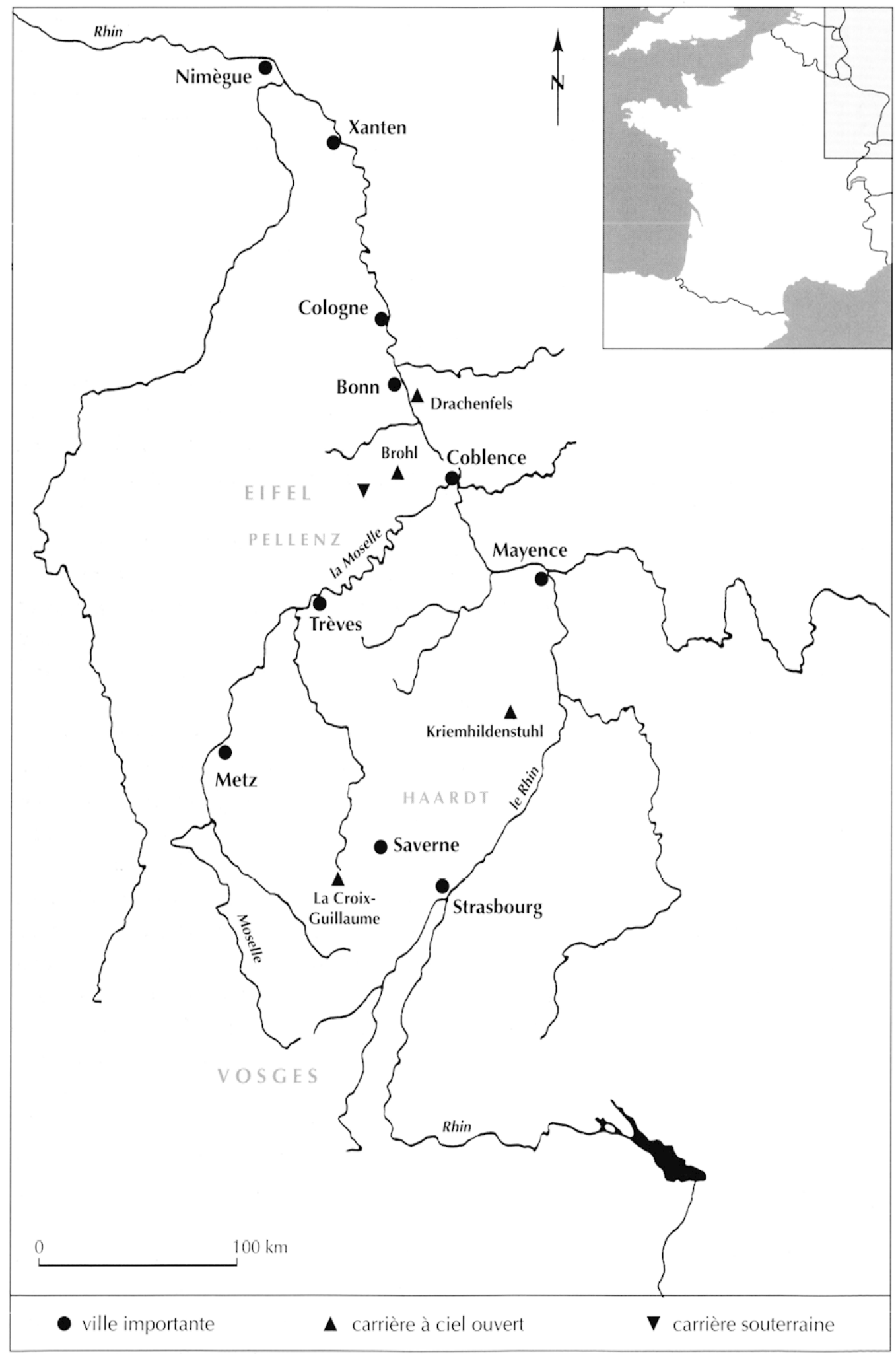

Fig. 118 - Carte des principales villes et sites d'extraction cités dans le texte (dessin Lukas, (NRS).

sent alors une expansion importante grâce à leurs nouvelles fonctions de sièges administratifs et militaires aux portes nord-est de l'Empire. Des projets urbanistiques de grande ampleur entrainent une forte demande en pierre de construction et par conséquent l'émergence et la prospérité d'un secteur de production comprenant toutes les étapes du travail de la pierre, depuis son extraction jusqu'à sa mise en ceuvre. Dans les régions mosel- 
lanes et rhénanes, qui constituaient des voies de circulation importantes, l'ouverture et l'activité de nombreuses carrières de pierre de taille s'insèrent dans ce contexte d'urbanisation soutenue. On connait aujourd'hui plusieurs grands centres d'extraction, qui furent implantés dans des gisements de roches extrêmement variés, afin de subvenir à de multiples besoins : parmi les plus importants, on trouve les exploitations de calcaire autour de Metz, les carrières de grès de la région de Trèves, leurs homologues dans les Vosges et le massif du Haardt, ainsi que les exploitations de tuf et de basalte du massif de l'Eifel (fig. 118).

Si l'intérêt scientifique pour les carrières du monde romain a augmenté et si le nombre d'articles et d'ouvrages spécialisés s'est multiplié au cours des deux dernières décennies, les recherches sur le terrain n'avancent qu'à petits pas et nous courons le risque que certaines carrières anciennes soient anéanties par des reprises d'extraction ou réutilisées comme décharge publique avant d'avoir pu être étudiées. Dans les régions rhénanes, les investigations archéologiques récentes sont rares et il faut souvent se contenter d'études anciennes. Celles menées par J. Röder dans les années cinquante et soixante méritent une attention particulière, même si elles se sont quasi exclusivement concentrées sur des structures visibles en surface ou dégagées fortuitement au cours de travaux d'exploitation modernes. Le faible nombre de carrières romaines connues à ce jour fausse notre perception de l'ampleur des activités d'extraction. C'est l'héritage architectural romain qui nous fournit une image plus réaliste des quantités de pierre extraites dans ces régions, et en particulier dans les bassins du Rhin et de la Moselle, qui permettaient l'acheminement des matériaux grâce à un réseau fluvial extrêmement dense. Il existe dans cette zone géographique un immense potentiel d'étude qui n'attend que l'engagement des archéologues intéressés par le domaine de la pierre. L'objectif de cette contribution est de faire le point de nos connaissances sur l'exploitation des gisements de roches dans ces régions frontalières au nord-est de l'Empire. Nous examinerons les études anciennes à la lumière des évolutions méthodologiques de la discipline, en les complétant avec des observations personnelles et des résultats de recherches récentes. Sans être en mesure de pouroir dresser une image exhaustive de l'exploitation romaine de la pierre dans ces régions, nous aborderons, dans une perspective générale, diffé- rents aspects de son organisation à partir des vestiges subsistants. Nous le ferons en retenant un nombre restreint de sites étudiés, mais inégalement répartis dans la zone géographique en question, puisque de nombreuses carrières encore visibles n'ont pas fait l'objet de datations.

\section{SOURCES ÉCRITES}

Si l'on trouve dans la littérature latine de brèves mentions des gisements et des roches, de leurs propriétés et emplois, des hommes intervenant dans les carrières et, plus rarement, des modes d'exploitation, rien de tel n'est attesté pour les régions du nord-est de la Caule et la Rhénanie romaine. Cette absence de texte reflète le faible intérêt que les auteurs latins portaient d'une manière générale aux lieux d'exploitation et en particulier aux travaux qui s'y déroulaient, exception faite des carrières de marbre, dont le matériau possédait un statut spécifique et bénéficiait, par conséquent, d'une attention particulière de la part des contemporains.

Dans la zone frontalière du nord-est, on trouve par contre de nombreux témoignages épigraphiques qui peuvent être des inscriptions sur les fronts de taille et sur les blocs extraits, ou des dédicaces sur des autels votifs provenant des comblements de carrières romaines. Ces documents parlent des hommes qui ont séjourné dans les carrières et dont un grand nombre étaient des soldats, détachés de leur unité militaire pour l'approvisionnement en matériaux de construction des villes de garnison rhénanes (fig. 119 et 120). Grâce à la mention des corps militaires, on est en mesure d'établir des jalons chronologiques pour les différentes phases d'exploitation; possibilité d'autant plus exceptionnelle qu'on ignore le contexte stratigraphique d'où proviennent la plupart des objets archéologiques issus de ces carrières.

Dédiés à Hercule Saxanus ou plus rarement à Jupiter, de nombreux autels découverts dans les comblements des galeries souterraines de la vallée de la Brohl, à une vingtaine de kilomètres au nord-ouest de Coblence (Rhénanie-Palatinat), témoignent de la présence ou du passage de soldats appartenant notamment aux légions I Minerva, VI Victrix, X (iemina, XV Primigenia, XVI (aallica, XXI Rapax ou à la XXII Primigenia (CIL, XIII, 7694 sqq.). Le cadre chronologique de l'exploitation militaire dans 


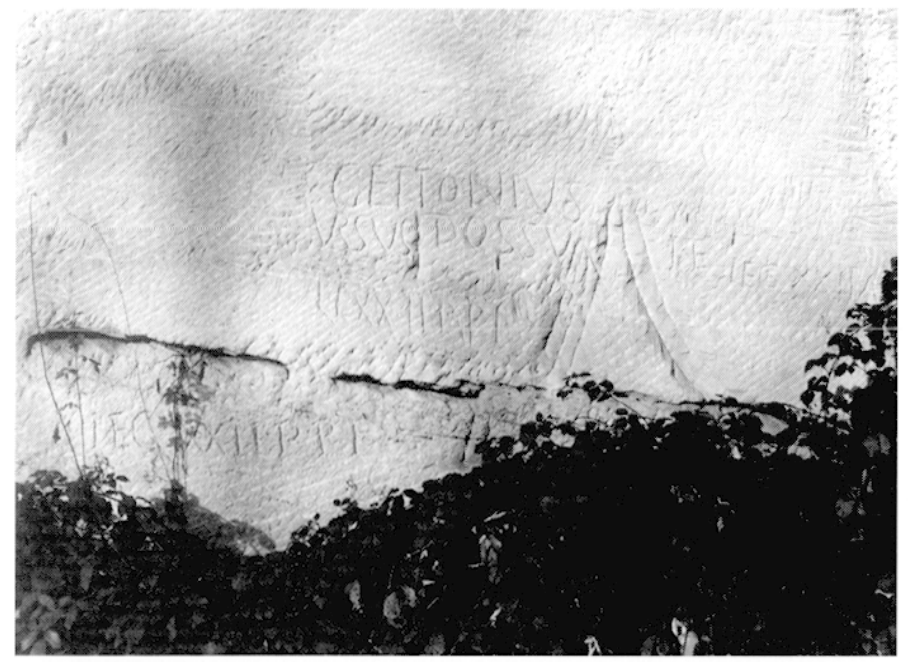

Fig. 119 - Inscription et marque technique à côté d'un défaut naturel sur un front de taille au nord-est de la carrière du Kriemhildenstuhl (Bad Dürkheim) : on lit les noms des trois carriers Gettonius, Ursus et Dossus, ainsi que celui d'une unité militaire, la legio XXII $\mathrm{P}$ (rimigenia) $\mathrm{P}$ (ia) $\mathrm{F}$ (idelis) ou $\mathrm{A}$ (ntoniniana) ou A(lexandriana) (photo P. Adam).

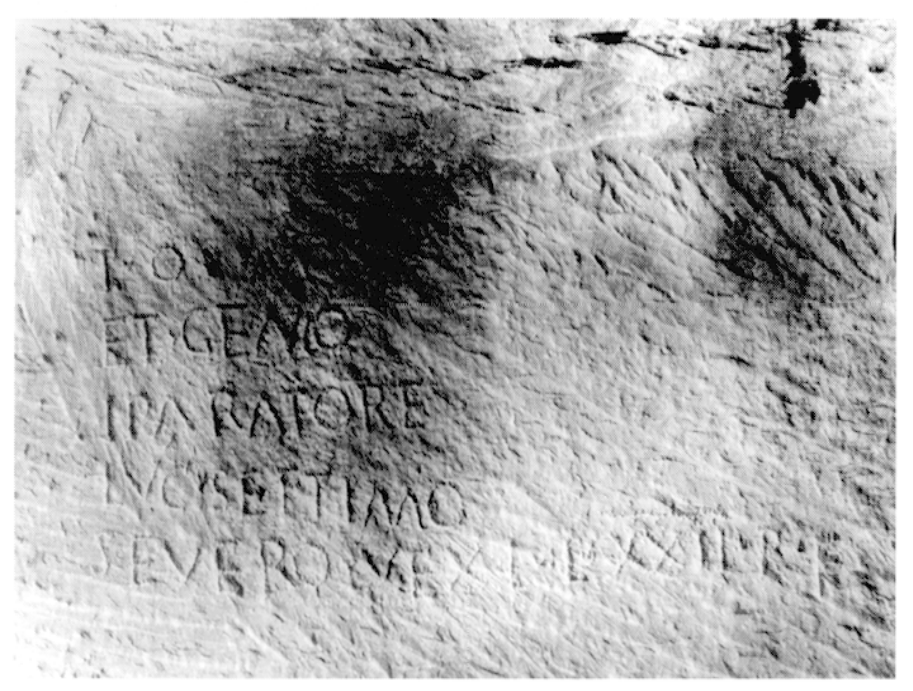

Fig. 120 - Inscription votive sur un front de taille au nord de la carrière du Kriemhildenstuhl dédiée par un détachement de la legio XXII P F à Jupiter, au genius loci et à l'empereur Lucius Septimius Sinerus (pholo P. Adlum).

la vallée de la Brohl, concentré sur les décennies séparant le règne de Claude de celui de Trajan, semble ainsi bien déterminé. Une activité antérieure n'est toutefois pas totalement à exclure. Selon J. Röder (1957, p. 229), l'absence de témoignages épigraphiques pour les époques suivantes n'est aucunement une preuve de l'abandon des carric̀res, mais plutôt le signe de l'efface- ment du monopole public de l'exploitation, car il semble que celle-ci se soit poursuivie jusqu'au Bas-Empire, probablement grâce à l'intervention de particuliers. Cette interaction étroite entre l'exploitation de la picrrc ct lc milicu militaire pendant le Ilaut-Empire se manifeste également dans les galeries souterraines de la Pellenz, une ancienne zone volcanique situce au sud de la vallée de la Brohl (Rhénanie-Palatinat). Les inscriptions sur des autels, des blocs extraits, des fronts de taille, ainsi que sur deux coins d'extraction en fer témoignent ici de la présence de légionnaires romains détachés de la VI" légion Victrix, stationnée à Neuss (Novaesium), de 70 à 105 de notre ère, de la $\mathrm{XXX}^{\circ}$ Ulpia, présente à Xanten (Vetera) à partir de 120 de notre ère ou de la Ir' Minerva (Röder, 1957, p. 228-229 ; Scholz, 1999, p. 9). Il en va de même pour le Kriemhildenstuhl, une carrière implantée dans un gisement de grès quartzifère à la bordure du Haardt, à une soixantaine de kilomètres au sud de Mayence (Rhénanie-Palatinat), où les sources épigraphiques font à plusieurs reprises mention de la legio XXI Primigenia Pia Fidelis, stationnée à Mayence (Mogontiacum) (fig. 119 et 120). À Reinhardsmunster, près de Saverne (Bas-Rhin), ce sont les soldats de la legio VIII Augusta (CIL, XIII, 5989) qui ont été chargés de l'extraction du grès vosgien. Des carriers appartenant à la même légion, associés à ceux de la XIX" Rapax, de la XIV“ Gemina Martia Vistrix et de la X" Gemina, ont travaillé sur les versants mosellans près de Mety, au $\mathrm{I}^{\mathrm{er}}$ et au début du $\mathrm{II}^{\mathrm{C}} \mathrm{s}$. de notre ère ( $C I L$, XIII, 4623-4625).

L'intervention du militaire dans la gestion et l'exploitation des carrières n'est pas un phénomène limité aux régions frontalières du nord-est de l'Empire, puisqu'elle se manifeste également à proximité du limes britannique où des sources épigraphiques provenant d'une série de carrières attestent que les soldats étaient chargés d'approvisionner le chantier du mur d'Hadrien (Dworakowska, 1983, p. 50-51 et 105). Cependant, malgré l'image suggérée par l'épigraphie d'une omniprésence militaire dans les carrières situées dans les territoires frontaliers, qui concentraient des forces militaires importantes, il ne faut pas sous-estimer le rôle des particuliers dans l'exploitation des ressources lithiques. Le phénomène des exploitants-militaires s'insère dans la problématique plus large de la propriété des carrières, qui se décline en questions sur la nature des propriétaires (État, communauté, particulier, etc.) et le statut 
juridique de l'exploitation (exploitation par le propriétaire ou système de concession), toutes choses qui n'ont à ce jour guère fait l'objet d'études ${ }^{90}$.

\section{SOURCES ICONOGRAPHIQUES}

Des représentations graphiques relatives à l'extraction sont connues dans les carrières romaines sous la forme d'illustrations abstraites ou figuratives incisées ou taillées dans les parois rocheuses. La Rhénanie nous fournit à ce propos quelques beaux exemples. L'un des plus connus, la gravure rupestre d'un carrier maniant son instrument de travail, provient d'une galerie souterraine de Kruft dans la Pellenz, Rhénanie-Palatinat (Röder, 1957, tabl. 21, fig. 1). De caractère très rudimentaire, ce graffito reproduit la silhouette d'un ouvrier qui tient à deux mains un outil à double tranchant et manche long, dont les dimensions sont toutefois exagérées par rapport à la taille humaine. Ce dessin rupestre constitue néanmoins un document d'autant plus important qu'il provient du contexte même des carrières ${ }^{91}$, et qu'il rend possible, malgré son tracé sommaire, des rapprochements avec des outils trouvés dans la même région (Röder, 1957, fig. 5-6), voire même avec l'escoude romaine connue en Gaule Narbonnaise (Bessac, 1996, p. 206-207, fig. 128-129). Il contribue ainsi à nos connaissances sur l'outil romain permettant le creusement des tranchées d'extraction.

Un nombre important de graffiti linéaires et figuratifs a été découvert sur les fronts de taille* et certains blocs du Kriemhildenstuhl lors des travaux archéologiques menés dans les années trente par F. Sprater. Répartis sur l'ensemble des parois rocheuses, une trentaine de gravures et reliefs rupestres subsistent encore sur place, dont certains portent des traces de couleur. Ces graffiti de qualité variable représentent à plusieurs reprises des têtes et figures humaines (fig. 121, $\mathrm{n}^{\mathrm{os}} 1$ et 3), des

90. J. Röder a tenté une analyse des problèmes de proprićté dans le contexte des carrières de basalte de Maven (Röder, 1956).

91. Des relicfs qui montrent vraisemblablement des carriers avec leur outil de trarail sont connus ailleurs, dans le monde méditerranéen, mais associés à d'autres contextes, comme par exemple le relief d'un carrier sur le Pont du Gard (Bessac, 1999b), une stèle d'Aliki (Sodini $e$ al., 1980), fig. 12) et un autel de Saint-Béat (Dworakowska, 1983, p. 116-117) chevaux (fig. 121, n'2), des oiseaux et d'autres animaux, des sexes masculins et féminins, ainsi que des motifs linéaires divers (fig. 121, n'4). Ils ont connu des interprétations divergentes, dont aucune n'apporte de conclusions satisfaisantes: si F. Sprater (1948) a voulu placer ces graffiti dans un contexte religieux indigène, J. Röder a proposé une lecture liée à la gestion de la carrière, en suggérant une corrélation entre symboles et équipes de travail (Röder, 1969). Selon lui, chaque groupe se désignait par un symbole précis et le reproduisait pour marquer son secteur de travail. L'apparition répétée d'un même signe sur des parois différentes témoignerait par conséquent, ainsi que le suppose cet auteur, d'un déplacement des équipes au sein de la carrière.

Si les graffiti du Kriemhildenstuhl restent encore objet de discussion, il est intéressant de signaler deux autres exemplaires trouvés dans l'aire d'extraction du Drachenfels, située à une dizaine de kilomètres au sud de Bonn, dans un gisement de trachyte quartzifère (Rhénanie du Nord-Westphalie). Bien que l'origine romaine des vestiges d'extraction reste à confirmer, une forte activité extractive est incontestablement attestée pendant le Haut-Empire par l'emploi du matériau même, diffusé jusqu'à Xanten et aux régions du Rhin inférieur. Les deux graffiti repérés sur des parois rocheuses de cette vaste zone d'extraction représentent un coq et un phallus (Röder, 1974, p. 520, fig. 11). Ce dernier motif figure également, parmi d'autres, dans la carrière de Saint-Boil (Saône-et-Loire), implantée dans des bancs massifs d'un calcaire tendre, à $25 \mathrm{~km}$ au sud-ouest de Chalon-sur-Saône (Monthel, Pinette, 1977, p. 55-61 et Monthel, supra, p. 89-120). Associés à des inscriptions de natures diverses sur les fronts de taille, les graffiti chalonnais rappellent fortement nos exemplaires rhénans: têtes humaines, oiseau, cavalier, phallus, etc.

S'agit-il d'un simple divertissement de la part des carriers, d'un moyen de se distraire d'un travail éprouvant et pénible, ou faut-il pousser plus loin l'interprétation des dessins et chercher leur explication dans un contexte administratif lié à la gestion de la carrière, ainsi que l'a proposé J. Röder? Il est impossible de fournir une réponse convaincante dans l'immédiat, et des études complémentaires, élargies à un cadre géographique plus large, sont indispensables. 


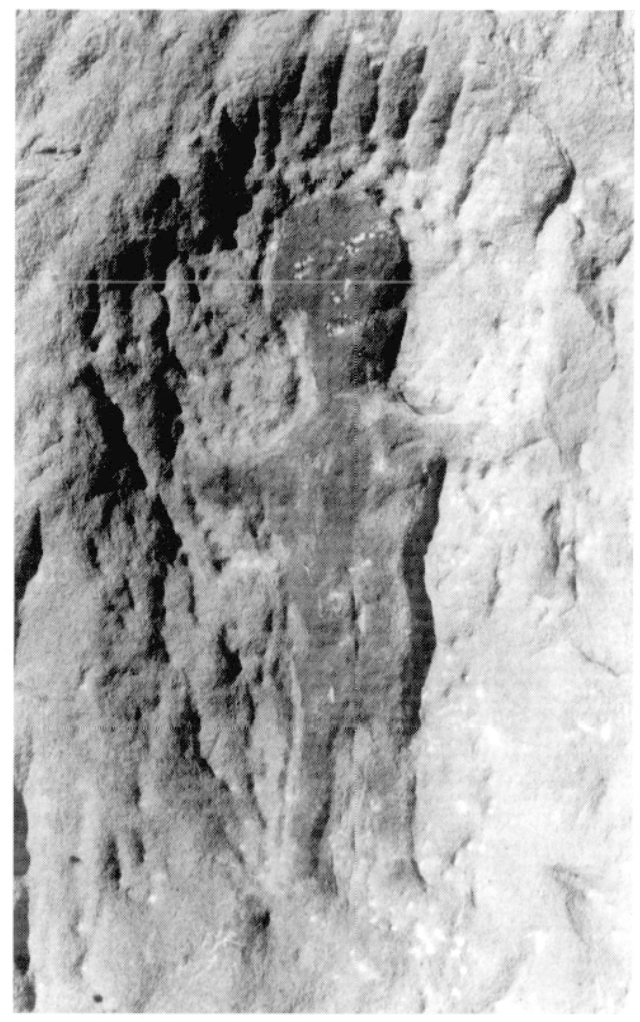

1

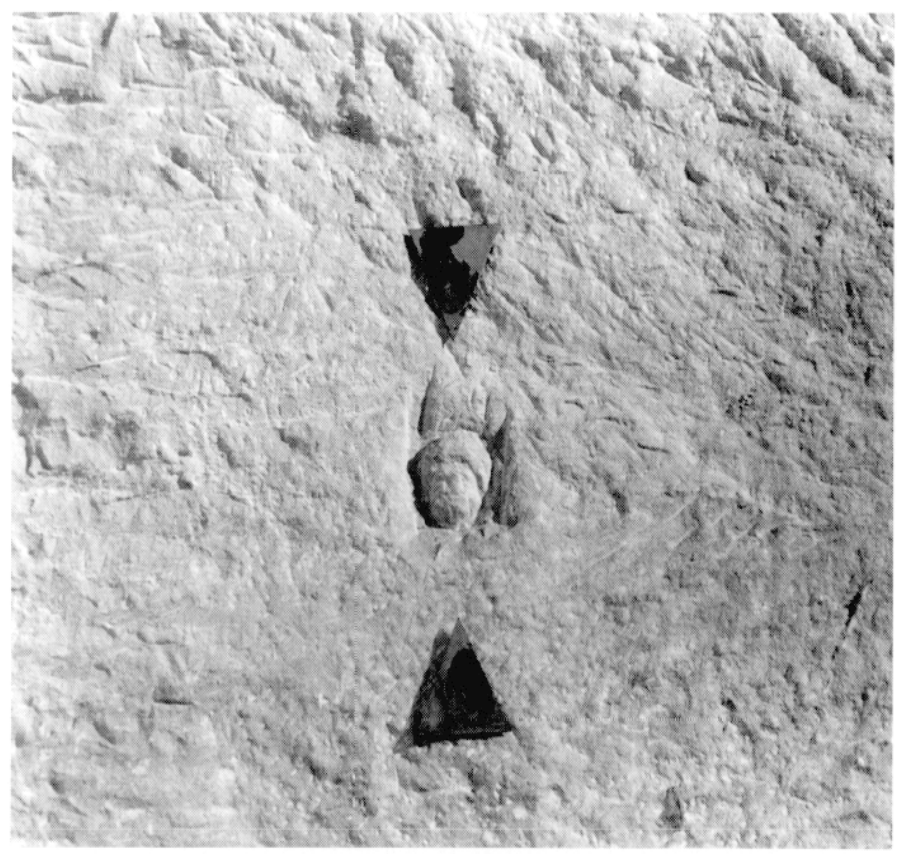

3

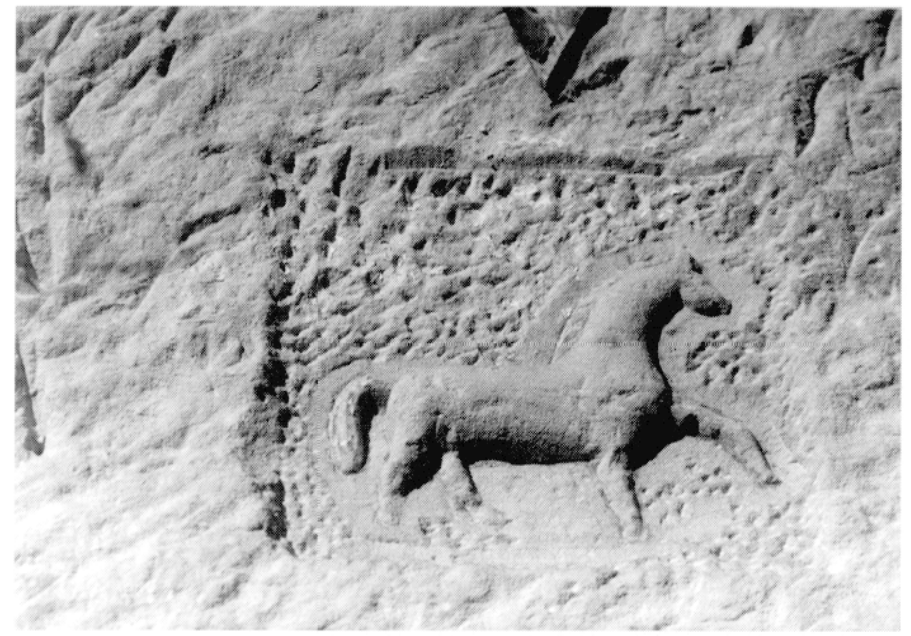

2

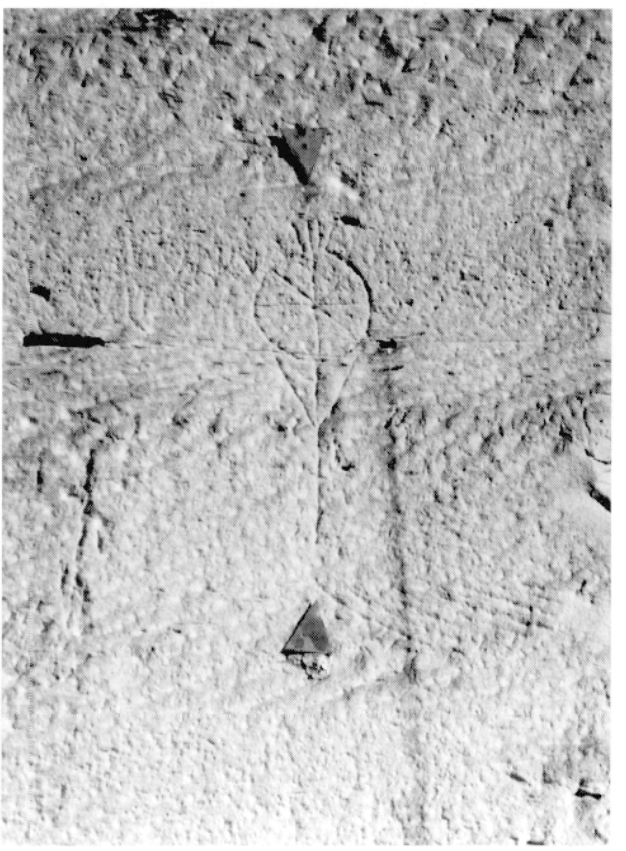

4

Fig. 121 - Carrière du Kriemhildenstuhl (Bad Dürkheim) : 1, relief d'un personnage (hauteur 35 cm environ) exécuté sur un front de taille au nord-est de la carrière; 2, relief d'un cheval (hauteur $25 \mathrm{~cm}$ environ) exéculé sur un front de laille au nord-est de la carrière; 3, visage humain (hauteur $10 \mathrm{~cm}$ envirom) taillé sur un front de taille au nord de la carriere; 4, signe abstrait (hauteur $75 \mathrm{~cm}$ environ) gravé sur un front de taille au nord-est de la carriere (photos P. Adam). 


\section{ORGANISATION ET MODES D'EXTRACTION}

L'emplacement des carrières romaines en Rhénanie répond à de nombreux critères qui ne se distinguent pas de ceux qui déterminent ailleurs le choix géographique d'une exploitation: l'existence d'un matériau qui réponde, en termes de qualité et de quantité, à des besoins spécifiques, l'accessibilité du gisement, ainsi que l'existence d'une voie de communication (terrestre, fluviale ou maritime), qui rende possible, de manière économique, le transport des blocs jusqu'aux chantiers de construction. Dans le nord-est de la Gaule et en Rhénanie, un réseau fluvial dense a permis de parcourir des distances souvent ćlevées entre carrières et lieux d'emploi, tout en minimisant les frais et les difficultés du transport par rapport à l'emprunt des voies terrestres. La possibilité d'un transport fluvial a même entrainé l'acheminement sur des distances inhabituclles d'une roche de qualité ordinaire, comme le montre l'exemple du trachyte du Drachenfels, qui a connu une diffusion audelà de Bonn et Cologne, respectivement distantes de 10 et $30 \mathrm{~km}$, afin d'atteindre, à plus de $100 \mathrm{~km}$, des débouchés situés dans la région du Rhin inférieur (Xanten et Nimègue), plus pauvres en roche de construction (Röder, 1974 et fig. 1). L'existence du Rhin au pied même du Drachenfels a sans doute influencé un tel choix, que la qualité moyenne du matériau aurait plutôt découragé ${ }^{92}$. Plus souvent, c'était la haute réputation d'une roche qui entraînait sa diffusion à longue distance, comme dans le cas du calcaire lorrain, extrait aux environs de Metz. et acheminé, sans doute par la Moselle, vers la vallée du Rhin, afin d'approvisionner en matériau de haute qualité les chantiers urbains (Bonn, Mayence, Nimègue, etc.).

Les travaux précédant et accompagnant l'ouverture d'une nouvelle carrière étaient multiples et variés, supposant une organisation complexe et l'intervention de spécialistes expérimentés. Le manque de sources littéraires et le fait que les vestiges résultant de ces travaux préliminaires aient généralement disparu au cours de l'extraction rendent difficile une étude de ces phases initiales de l'exploitation. En revanche, les

92. Les cristaux de sanidine empechaient toute taille profonde de la surface rocheuse et limitaient par conséquent l'utilisation du matériau. techniques de l'extraction proprement dite sont lisibles souvent jusqu'au détail par les traces qu'elles ont laissées. Dans le nord-est de la Gaule et en Rhénanie, extraction à ciel ouvert et travail en galeries souterraines étaient pratiqués. Ils le furent même conjointement au sein d'une même carrière. Si le choix pour l'un ou l'autre type d'extraction était principalement dicté par les conditions géomorphologiques du terrain (la disposition du banc de roche, le pendage du terrain, etc.), il ne faut pas sous-estimer les contraintes économiques dans cette démarche. Une comparaison des carrières romaines dans ces régions semble montrer une préférence pour l'extraction à ciel ouvert - s'il ne s'agit pas ici d'une réalité fausséc par le fait que des vestiges en surface attirent plus l'oxil que les espaces souterrains, habitucllement comblés par des déblais et occultés par la végétation qui rend invisibles les accès aux aménagements du sous-sol. Sur le terrain, les carriers ont dû chercher dans chaque cas individuel le meilleur compromis entre les conditions naturelles, les moyens financiers et la technique d'extraction la plus adaptée.

L'exploitation du tuf dans la Pellenz s'est pratiquée dans un premier temps à ciel ouvert, sous forme de paliers sur les versants d'une petite vallée entre Kruft et Plaidt, où la rivière avait déjà mis à nu une première couche de tuf exploitable (Lehner, 1921 ; Röder, 1957, fig. 22.1). ('e n'est que dans un deuxième temps qu'une augmentation de l'épaisseur de la découverte, la couche de roche supérieure, inutilisable en tant que pierre de taille, a poussé les carriers à pénétrer horizontalement, par des galeries, dans l'intérieur du coteau pour poursuivre l'extraction en milieu souterrain, tout en laissant en place la découverte dont l'enlèvement aurait constitué une perte de temps.

Un travail à l'air libre a été préféré pour l'exploitation du grès dans le Kriemhildenstuhl, où une découverte peu épaisse a incité les Romains à implanter une carrière en paliers sur le flanc du coteau, en partant des bancs supérieurs, pour ensuite approfondir l'exploitation d'une vingtaine de mètres environ, sans recourir à la solution adoptée dans la Pellenz. (fig. 122). La faible épaisseur de découverte, qui ne dépasse pas $1 \mathrm{~m}$ à certains endroits, a en effet réduit les travaux préliminaires d'ouverture de la carrière, ainsi que la quantité des déblais qui constituent un risque permanent de comblement précoce du site. 


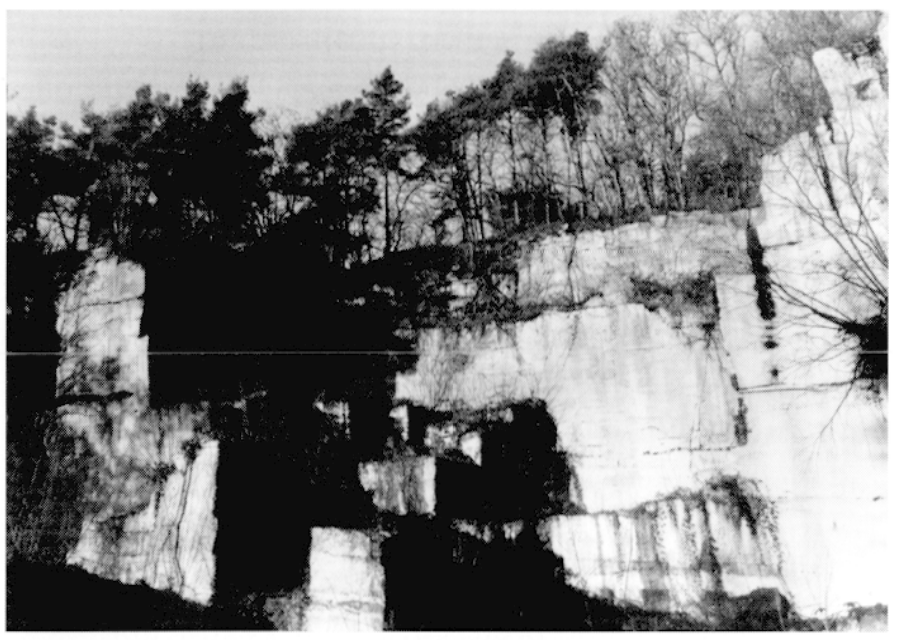

Fig. 122 - Carrière du Kriemhildenstuhl (Bad Dürkheim) : à l'arrière-plan, le front nord-ouest de la carrière; au milieu, plusieurs secteurs de travail sur des niveaux différents; $\grave{a}$ droite, les parties les plus profondes de l'excavation (photo P. Adam).

L'exploitation gallo-romaine du grès vosgien s'est également effectuée à l'air libre, comme en témoignent plusieurs exploitations, de taille modeste, sur le plateau de La Croix-Guillaume, dans les Vosges mosellanes (cf. Heckenbenner et Meyer, supra, p. 145-154). Un terrain quasi horizontal a favorisé ici l'aménagement d'une extraction en fosse, qui s'est développée verticalement à partir d'une surface plane, à l'intérieur de la masse rocheuse, et dont l'accès était assuré par une rampe inclinée. Le pourtour du plateau était entamé par d'autres carrières d'extension plus modeste.

Un mode d'exploitation particulier s'observe dans l'aire d'extraction du Drachenfels (Rhénanie du NordWestphalie) implantée dans un gisement de trachyte. Des vestiges attribués à l'époque romaine y ont été examinés par J. Röder, qui les a publiés dans un rapport préliminaire (Röder, 1974). Ils se situent aux abords nord du massif, en des lieux nommés Rüdenet et Drachenburgpark, où l'on trouve d'énormes rochers, qui se sont détachés naturellement du substrat et ont glissé sur le coteau depuis des endroits plus élevés jusqu'à leur emplacement actuel. Ces "mers rocheuses" forment une ressource lithique disponible en surface et facilement exploitable, dans la mesure où il suffisait de " retailler " ces blocs aux dimensions souhaitées, sans être obligé de concentrer l'extraction à un endroit précis et d'investir dans l'aménagement d'une carrière en profondeur ${ }^{93}$. Grâce à leur taille imposante, ces rochers pouvaient fournir des blocs de grand appareil dont l'extraction faisait surtout appel à des coins métalliques et à la technique du clivage ${ }^{*}$, beaucoup plus économe en temps que l'emploi du pic d'extraction.

\section{LE KRIEMHILDENSTUHL : ORGANISATION ET FONCTIONNEMENT D'UNE CARRIÈRE ROMAINE}

Les besoins importants liés aux projets urbanistiques ont entrainé production et extraction à grande échelle. En parallèle il existait sans doute des exploitations de moyenne et petite taille, gérées par des entreprises ou des particuliers, comme à Mayen probablement, où l'exploitation romaine du basalte s'effectuait sur des parcelles de faible étendue. Les exploitants n'y disposaient pas de moyens suffisants pour répondre à une production de masse, mais devaient par contre jouer un rôle non négligeable sur le marché local de la pierre (Röder, 1956). D'autres sites ont connu une exploitation intense, nécessitant une organisation complexe. C'est le cas de la carrière du Kriemhildenstuhl, située en bordure du massif du Haardt, dans laquelle l'extraction du grès s'effectuait au Haut-Empire dans plusieurs carrières indépendantes. Les vestiges d'extraction montrent comment les Romains ont procédé afin d'assurer, de manière constante et régulière, l'approvisionnement d'un, voire plusieurs chantiers de construction ${ }^{94}$.

L'exploitation a suivi une progression vers l'intérieur du coteau, sous forme de paliers, en plusieurs sections constituant, au cours de l'exploitation, des secteurs de travail autonomes et parallèles (fig. 122). L'analyse du contexte lithostratigraphique et du plan de la carrière montre que les carriers romains ont été obligés de rétrécir la surface de l'exploitation au fur et à mesure de la progression, afin de contourner des lithoclases de taille importante qui limitaient l'exploitation latéralement et empêchaient la progression dans certaines directions (fig. 123). Cette démarche a entraîné une subdivision en

93. D'autres " mers rocheuses ", où de grands blocs érodés ont ćté mis en exploitation dans l'Antiquité, existent, notamment dans des affleurements de granite d'Assouan (Röder, 1965) et dans l'Odenwald en Allemagne (Röder, 1959)

94. Le volume total de roche extraite s'élève, selon Röder, approximativement à $20000 \mathrm{~m}^{3}$ brut (Röder, 1969, p. 111). 
ressauts successifs des fronts de carrière latéraux, tandis que le front principal a plus ou moins gardé son allure linéaire. Le sol de carrière, qui reste partiellement enfoui sous des déblais, met en évidence quinze plates-formes de travail indépendantes, en forme de rectangle ou de trapèze, qui sont réparties sur des niveaux différents, sans homogénéité dimensionnelle, puisque le secteur le plus grand s'étend sur environ $400 \mathrm{~m}^{2}$, tandis que le plus petit ne couvre que $53 \mathrm{~m}^{2}$. Les limites de ces aires d'extraction coincident en plusieurs endroits avec les lithoclases qui parcourent la masse rocheuse et imposèrent une orientation stricte de l'exploitation, afin d'éviter des pertes de matériau trop importantes (fig. 123). Cette subdivision spatiale de la carrière est également rappelée par une inscription rupestre évoquant le terme angulus, qui semble faire référence à un angle de front de taille délimitant de manière naturelle une plate-forme de travail ${ }^{95}$. Dans chacune des \%ones d'extraction ainsi circonscrites, les carriers romains ont appliqué sur le sol de carrière des canevas d'extraction réguliers et orthogonaux. En étudiant les empreintes de blocs dans trois secteurs exploités, J. Röder a pu distinguer sept groupes métrologiques différents, dont le plus grand comprend des éléments mesurant $300 \mathrm{~cm} \mathrm{x} 140 \mathrm{~cm} \mathrm{x} 60 \mathrm{~cm}$ et le plus petit des blocs de $120 \mathrm{~cm} \times 110 \mathrm{~cm} \times 60 \mathrm{~cm}$ (Röder, 1969, p. 123).

La pratique d'une subdivision spatiale de l'aire d'extraction amène à s'interroger sur les conséquences qui en résultaient pour le déroulement du travail dans la carrière. Par cette stratégie, les responsables ont sans doute cherché à augmenter le nombre de carriers chargés des diverses opérations de travail dans chacun des secteurs. L'extraction simultanée de plusieurs blocs, qui pouvaient être de modules différents, était ainsi

95. Cf. Röder, 1969, p. 126, nº 2 :

OSTILI GENIALI

ANGVLVS QVIN

PVRPVRIONIS

L'interprétation de cette inscription, qui est encore bien lisible sur un front de taille, est sujette à controverses dans la mesure où J. Röder (1969, p. 126) propose de lire la première ligne comme [HJOSTILIII] (EENALIIS], désignant selon lui une centuria on une vexillatio, contrairement à F. Sprater (1948, p. 45 et 48) qui relie le mot angulus à Hostilis Cienialis. J. Röder considère en outre que le mot QVIN ne correspond pas au numéro d'ordre d'un angulus, mais devrait être lu plutôt comme le nom d'un persomnage : QLIN/TIJ ou Q[IN/TILII], responsable d'un secteur. Notons toutefois que cinq secteurs semblent avoir éte en activité simultanément. Cf. le terme anglares inscrit dans la carrière bourguignonne de Saint-Boil (Monthel, 1984, p. 175).

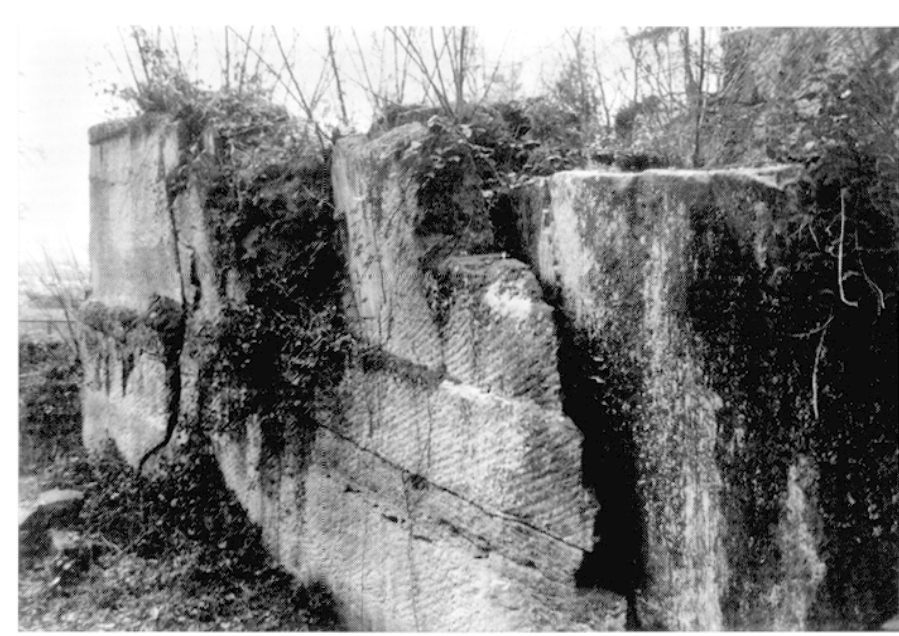

Fig. 123 - Lithoclase parcourant la carrière du Kriemhildenstuhl (Bad Dürkheim) en divertion nord-ouest/sud-est et marquant la limile entre plusimurs secteurs de travail (photo P. Adam).

réalisable et un approvisionnement constant des chantiers de construction garanti. La rigidité du canevas d'extraction permettait une production de masse, mais pouvait entraîner des pertes de matériau lorsqu'il croisait des défauts naturels imperceptibles auparavant. L'organisation en secteur pose également la question du nombre de carriers travaillant dans les différentes zones de la carrière et dont certains ont immortalisé leur nom sur les fronts de taille (fig. 119). L'absence d'une étude suffisamment détaillée des données techniques et d'une évaluation qualitative du travail, qui pourrait amener à la distinction de mains individuelles, nous empêche d'avancer sur cette question. Si J. Röder (1969, p. 123-124) avance l'hypothèse d'une présence de quatre carriers par secteur en moyenne, aucun critère scientifique ne permet à l'heure actuelle de confirmer ou d'infirmer cette proposition ${ }^{9}$.

\section{TECHNIQUES D'EXTRACTION}

Aucune source grecque ou latine décrivant en détail les techniques d'extraction utilisées dans les carrières romaines ne nous est parvenue. Les recherches archéologiques doivent donc principalement s'appuyer sur les traces conservées dans les carrières et résultant des

96. La distinction de mains individuclles suppose toutefois un bon ćtat de conservation des traces et une solide expérience de leur lecture. Elle laisse en tout état de cause une marge d'incertitude non négligeable. 
diverses opérations de travail. En ce qui concerne le nord-est de la Gaule et la Rhénanie, il nous faut malheureusement constater une fois de plus que nos connaissances sont loin d'être satisfaisantes puisque les études sur les données techniques font quasiment défaut. De nombreuses questions restent donc ouvertes et des précisions ne pourront être apportées qu'après de nouveaux engagements archéologiques sur le terrain.

\section{LE KRIEMHILDENSTUHL : UNE EXTRACTION EN LIT}

La carrière de grès du Kriemhildenstuhl (RhénaniePalatinat) constitue un des rares sites de Rhénanie dont les aspects techniques ont été abordés par J. Röder (1969). Ce chercheur a mené en parallèle des observations ethno-archéologiques sur l'extraction traditionnelle du tuf dans la région de l'Eifel ${ }^{97}$. Dans le Kriemhildenstuhl, les tranchées d'extraction* abandonnées à divers stades d'avancement nous renseignent sur l'opération de havage telle qu'elle fut pratiquée par les carriers antiques (fig. 124). Elles ne se distinguent pas fondamentalement de ce que l'on observe ailleurs, lorsque l'extraction cherchait à suivre les bancs horizontaux de la roche. Le bloc était délimité sur ses faces latérales par des tranchées, puis on le détachait au niveau de sa base. Lors du creusement de la tranchée, le carrier a procédé en plusieurs passes parallèles, c'est-à-dire qu'il a aligné ses coups successivement le long de petites rainures faiblement espacées les unes par rapport aux autres sur toute la largeur de la tranchée (fig. 124, nº 3 ). À partir des observations ponctuelles, on recense dans ce contexte trois ou quatre passes parallèles à l'ouverture des tranchées qui se réduisent à deux sillons au fond. Ce rétrécissement des tranchées s'insère évidemment dans un contexte économique où l'on cherche à réduire le temps de l'opération de havage, tout en maintenant une production maximale. Pour éviter un rétrécissement trop fort de la tranchée en profondeur, provoqué par des sillons latéraux sur les parois qui " guident » les coups dc pic, les carriers ont toutefois dû élargir les tranchées

97. Sans nier l'intérêt d'une étude comparative des techniques d'extraction d'époques différentes, nous devons néanmoins regretter un certain manque de précision de la part de ce chercheur lorsquil s'agit de distinguer les observations réalisées auprès des carricrs modernes et les données techniques relevées dans la carrière romaine. profondes à leur ouverture. Pour la finition du fond des tranchées, au niveau des angles, il était courant de changer de direction et de poursuivre le creusement du côté opposé, un phénomène qui s'observe grâce aux impacts visibles sur les fronts de taille*. Il est difficile d'établir une base statistique des caractéristiques dimensionnelles des tranchées d'extraction du Kriemhildenstuhl, puisque la carrière n'est que partiellement dégagée et qu'une faible quantité de vestiges a été étudiéc. J. Röder a toutefois comparé les tranchées romaines avec leurs homologues modernes dans le tuf de l'Eifel et a conclu qu'on retrouve une relation constante entre leur largeur à l'ouverture et leur profondeur, de sorte qu'une profondeur de $60 \mathrm{~cm}$ impose une largeur à l'ouverture de $11 \mathrm{~cm}$, et que chaque approfondissement de la tranchée d'environ $10 \mathrm{~cm}$ implique un élargissement de celle-ci de $1 \mathrm{~cm}$ (Röder, 1969, p. 119).

Pour le creusement des tranchées, les carriers du Kriemhildenstuhl ont employé divers pics d'extraction, dont plusieurs exemplaires ont été découverts lors du dégagement du site et se trouvent actuellement au Heimatmuseum de Bad Dürkheim en Rhénanie-Palatinat (fig. 125). Pour l'archéologue, l'identification de différents outils d'extraction au sein d'une même carric̀re doit aussi passer par une étude détaillée des impacts visibles au fond des tranchées, dont les caractéristiques dimensionnelles doivent être étudiées de manière comparative. Malheureusement, le fouilleur ne s'exprime pas à ce sujet en détail, ce qui rend problématique d'associer les instruments, trouvés hors contexte stratigraphique, aux empreintes visibles dans les tranchées d'extraction, même si mes propres observations, très partielles, m'amènent à lier les deux.

Les sillons produits lors de l'impact sur les parois de la tranchée dépendent d'un effet combiné entre la dureté de la roche, l'efficacité de l'extrémité active de l'outil et l'énergie d'impact de celui-ci, ainsi que l'angle d'attaque (fig. 124, n's 1 et 126). L'examen de leur régularité constitue un moyen d'évaluer la qualité du travail. Leur longueur varie fortement dans le Kriemhildenstuhl et se situe sclon J. Röder (1969, p. 119) entre 2 et 8 cm ${ }^{98}$.

98. L'affirmation de J. Röder (1969, p. 118) que les fronts romains montrent généralement des sillons courts et épais à cause de l'utilisation de pics d'extraction trapus, plus tard abandonnés en faveur d'outils plus élancés, me parâit délicate et ne peut guère être généralisée, d’autant plus que des instruments très allongés sont bien connus dans le monde romain, et en particulier en (jaule (Röder, 1957, fig. 6 ; 


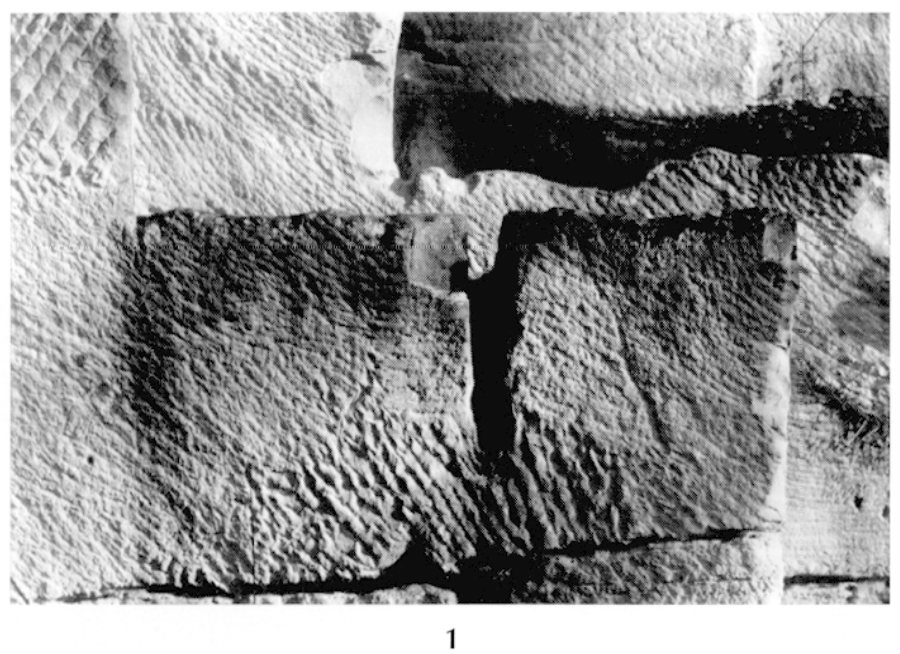

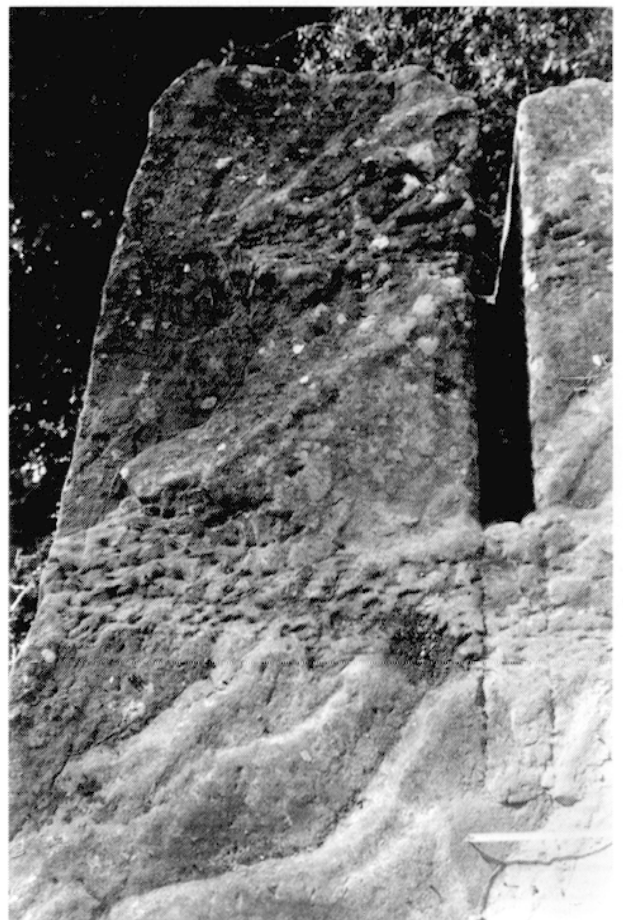

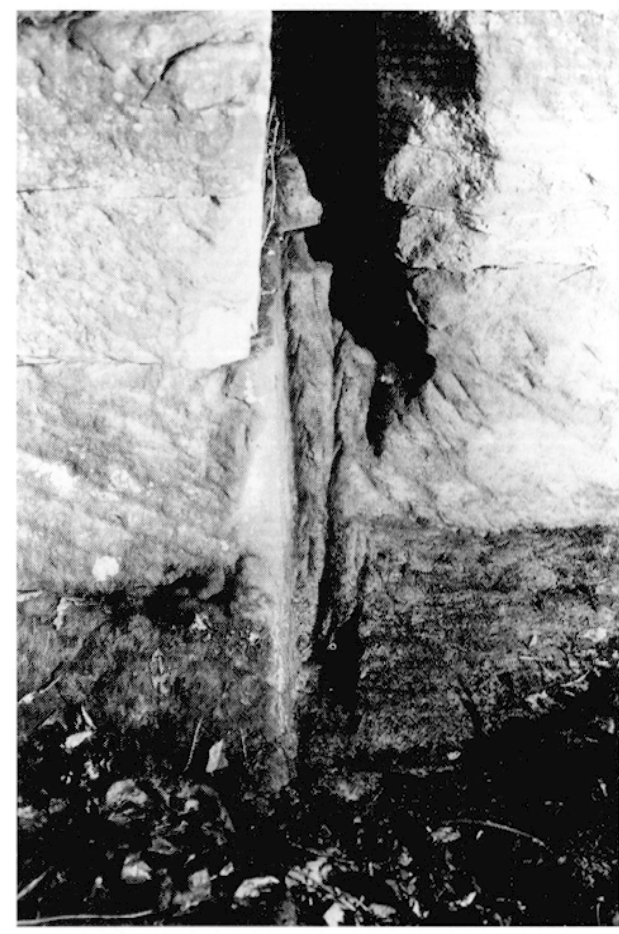

3

Fig. 124 - Carrière du Kriemhildenstuhl (Bad Dürkheim) : 1, tranchée d'extraction inachevée et sillons produits par l'outil lors du creusement des tranchées dans le secteur nord-est de la carrière; 2 , tranchée d'extraction inachevée avec le tracé préparatoire réalisé au pic dans la partie nord-est de la carrière; 3, extrémité d'une tranchée d'extraction réalisée dans le secteur central de la carrière, en trois passes réduites à deux dans la partie inférieure (largeur à l'ouverture: $14 \mathrm{~cm}$; largeur au fond : $8 \mathrm{~cm}$ ) (photos $P$. Adam).

L'observation des fronts de taille révèle une autre particularité qui répond aux difficultés rencontrées lors du creusement d'une tranchée située dans le prolongement

Bessac, 1996, p. 206-207). Soulignons que, contrairement aux empreintes figurant au fond des tranchées, les sillons qui recouvrent les fronts de taille reflètent en première ligne une technique de travail et permettent de distinguer des professionnels de capacités différentes, plutôt qu'une allure physique particulière de l'outil d'extraction. vertical d'une paroi haute. Cette dernière restreint naturellement l'espace nécessaire à un maniement aisé de l'outil d'extraction. Pour diminuer le risque d'un frottement violent des mains contre le front de taille, les carriers ont opté pour deux solutions au niveau des fronts de carrière déterminant la limite de l'exploitation (fig. 127). L'une des méthodes consistait à décaler la tranchée d'extraction en retrait du front, légèrement 

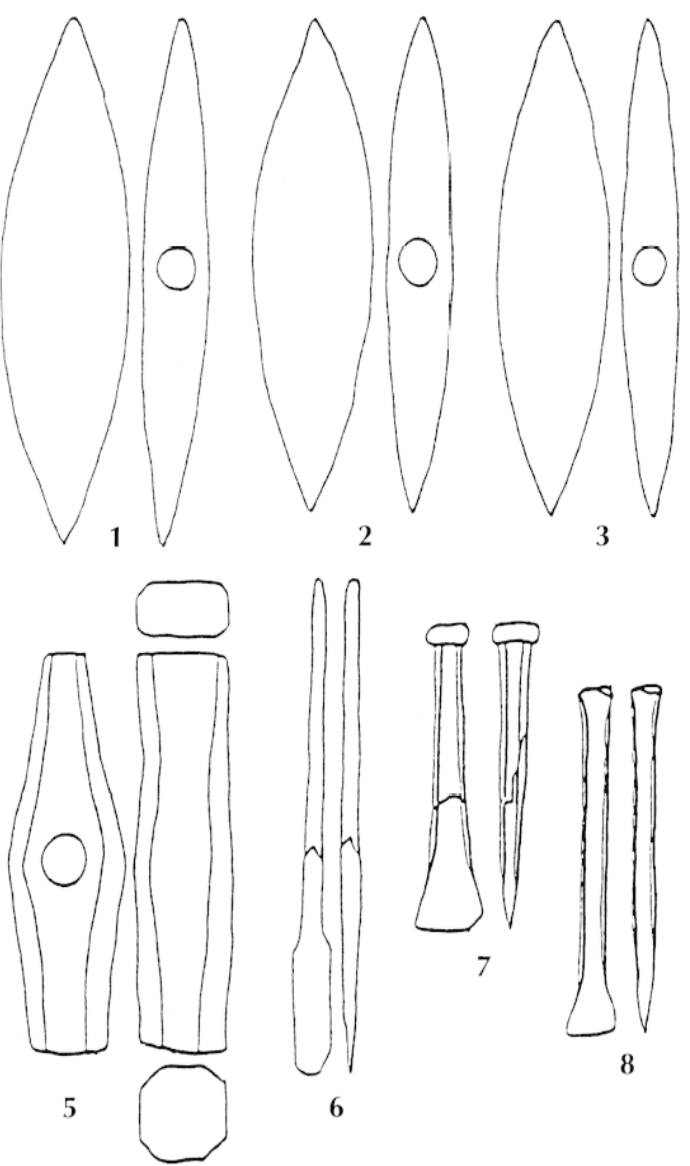

\begin{abstract}
6
\end{abstract}

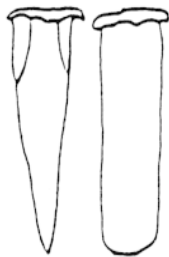

10

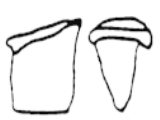

14

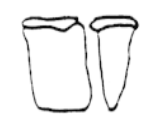

15

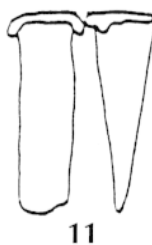

11

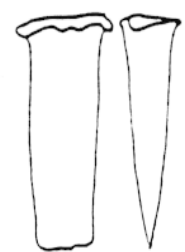

16
8
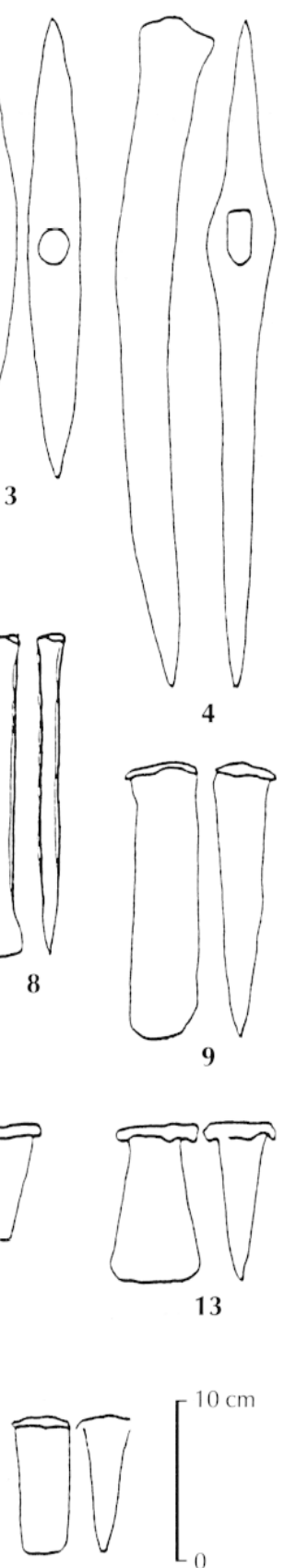

17
Fig. 125 - Outils de carrier provenant de la carrière du Kriemhildenstuhl, Bad Dürkheim (dessin modifié d'après Röder, 1969).

vers l'intérieur de l'aire d'extraction, afin de s'écarter sensiblement de la paroi verticale du front de taille. $\mathrm{Au}$ fur et à mesure d'une progression en profondeur, les parois se trouvaient par conséquent marquées sur leur hauteur par des ressauts plus ou moins accusés, tandis que la surface d'extraction diminuait sensiblement. La seconde technique, pour laquelle on note une certaine préférence dans le Kriemhildenstuhl, consistait à creuser

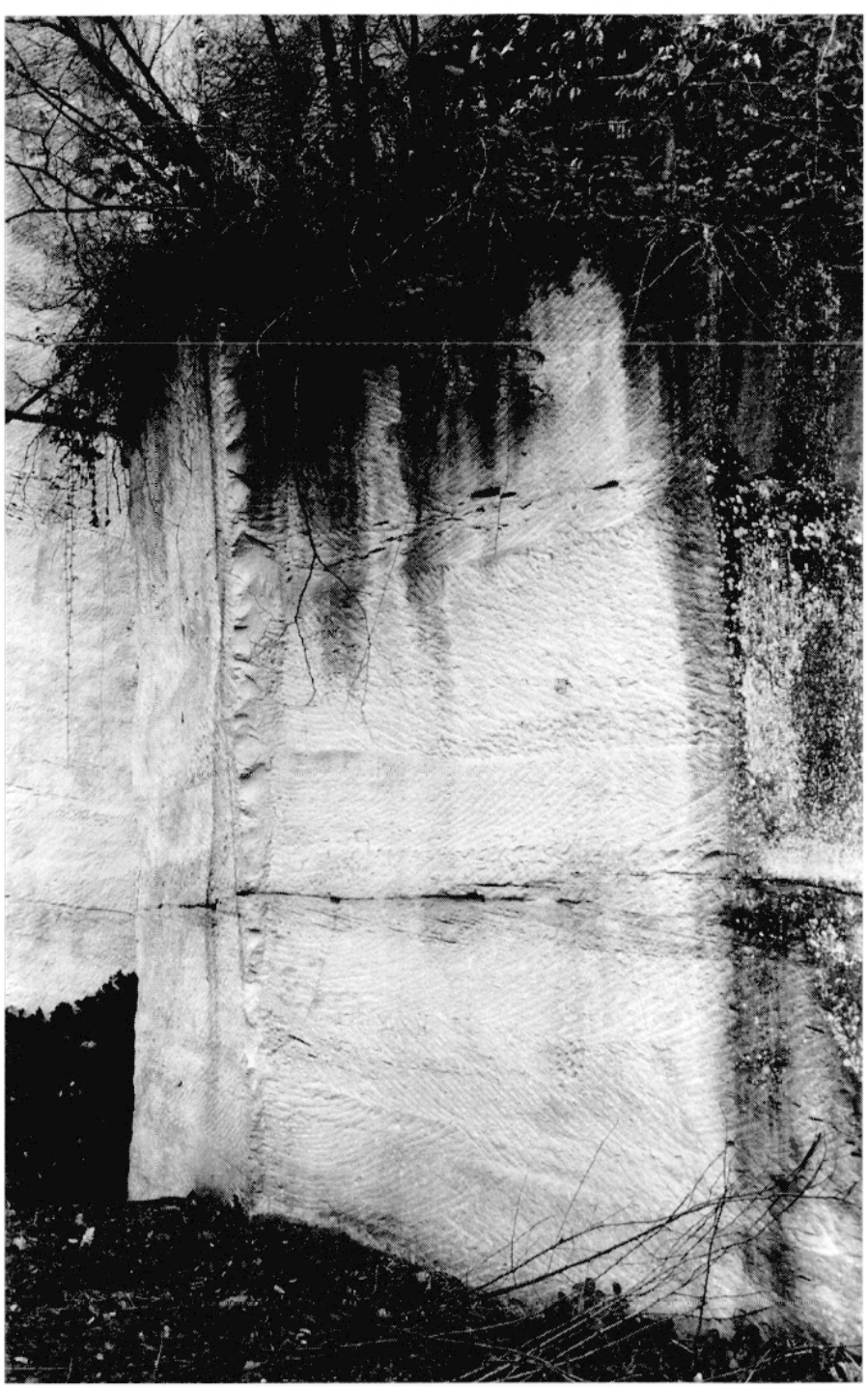

Fig. 126 - Front de taille dans la partie nord de la carrière du Kriemhildenstuhl (Bad Dürkheim) montrant la profondeur des tranchées et la régularité du travail. Le fronl porle une inscription. votive à mi-hauteur et une mention de la legio XXII dans sa partie. inférieure (photo P. Adam).

la tranchée d'extraction en biais, légèrement vers l'intérieur de la paroi rocheuse. Lors de la reprise de l'extraction sur un niveau inférieur, les carriers ont pratiqué le même schéma, en inclinant toujours plus les tranchées d'extraction, afin de disposer d'un espace de travail suffisant. La paroi était ainsi sous-creusée et prenait une inclinaison de plus en plus forte, laissant sensiblement augmenter la surface de l'aire d'extraction ${ }^{99}$.

99. Phénomène courant dans des carrières d'époques diverses, les deux solutions s'étudient de manière exemplaire dans les exploitations calcaires de la Maréotis en Égypte (Röder, 1967). 
Les difficultés rencontrées lors de l'aménagement d'une tranchée à proximité immédiate d'un front de taille amènent à une autre réflexion concernant l'existence de gauchers et droitiers au sein de la carrière. L'espace disponible pour l'opération de havage étant restreint à ces endroits, il existait des configurations plus ou moins favorables pour un gaucher ou pour un droitier, sachant qu'un droitier, qui guide son outil avec la main droite placée devant la gauche sur le manche, a tendance à frapper légèrement de la droite vers la gauche, et un gaucher en sens inverse. La préférence pour une main n'implique sans doute pas obligatoirement un caractère inné de droitier ou gaucher, mais chaque carrier garde néanmoins une préférence latérale et ne change qu'avec difficulté de côté, bien que ce changement soit, semblet-il, généralement plus facile pour un gaucher.

La confection d'une tranchée délimitée sur son côté gauche par une paroi verticale favorisait le travail d'un droitier qui courait moins le risque d'un frottement violent des mains contre la surface rocheuse qu'un gaucher, et inversement. À condition que l'aire d'extraction leur en offre la possibilité, les carriers, qui n'étaient pas habitués à guider l'outil avec l'autre main, changeaient sans doute de position par rapport à la paroi et creusaient la tranchée à partir de la direction opposée. Mais ce n'était pas toujours possible (par exemple si la tranchée se terminait au pied d'un front de taille* haut), et la présence de carriers gauchers et droitiers était par conséquent très utile au fonctionnement d'une exploitation, ce qui était encore plus vrai pour le creusement des tranchées verticales et des encoignures lors d'une extraction des blocs sur la hauteur du front, comme elle fut pratiquée dans les milieux souterrains de l'Eifel (voir infra). En ce qui concerne le Kriemhildenstuhl, J. Röder (1969, p. 120-121) a constaté une prédominance de droitiers, tandis que la répartition semble en nette faveur des gauchers dans d'autres carrières romaines ${ }^{100}$.

Pour séparer les blocs du substrat, les carriers du Kriemhildenstuhl ont quasi exclusivement pratiqué un détachement horizontal, technique la plus courante pour provoquer la rupture du bloc du substrat (fig. 128 et 129). Elle se pratiquait parallèlement au litage de la

100. Dans les carrières souterraines de tuf de l'Eifel la proportion droitiers-gauchers s'élève, selon J. Röder (1957. p. 260), à environ un pour deux à certains endroits. Une prédominance de gauchers a été également signalée par J.-C. Bessac (1996, p. 296) dans le contexte des exploitations romaines du Bois des Lens (Gard).

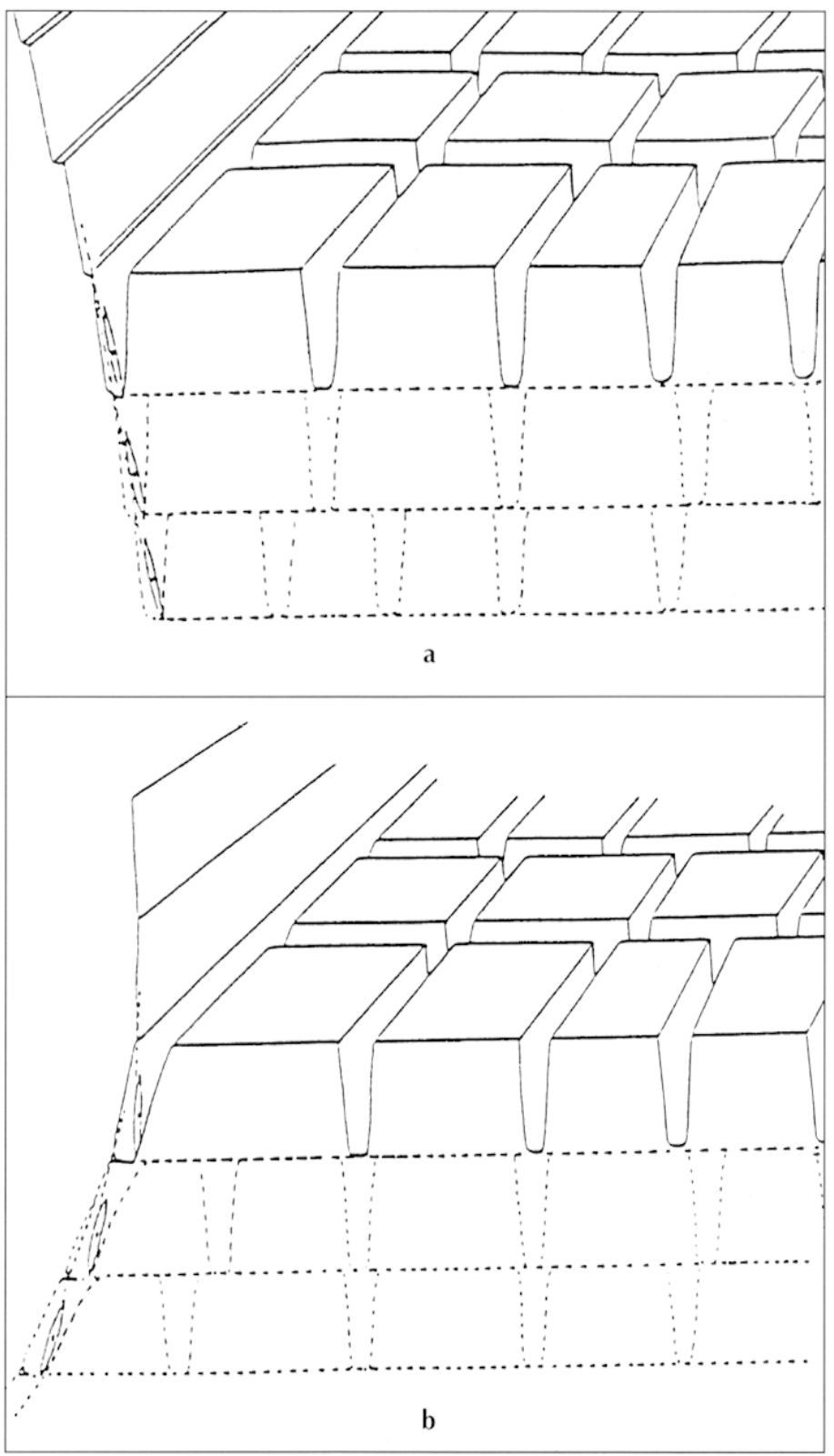

Fig. 127 - Principe du creusement de tranchées d'extraction au pied d'un front de taille élevé : $a$, en ressauts; $b$, en biais vers l'intérieur du front (dessin modifié d'après Röder, 1967).

roche, au niveau de la face inférieure du bloc. L'existence de joints de stratification a facilité l'action des coins dans le gisement du Kriemhildenstuhl. Les coins utilisés dans ce contexte étaient en fer, comme en témoignent des exemplaires de tailles différentes retrouvés dans la carrière (fig. 125, nos 9-17). Quant à l'outil ayant servi au creusement des emboîtures, J. Röder (1969, p. 121) fait l'hypothèse qu'un ciseau ait pu intervenir, ainsi qu'un pic d'extraction. On peut néanmoins douter de l'utilisation du pic pour cette 


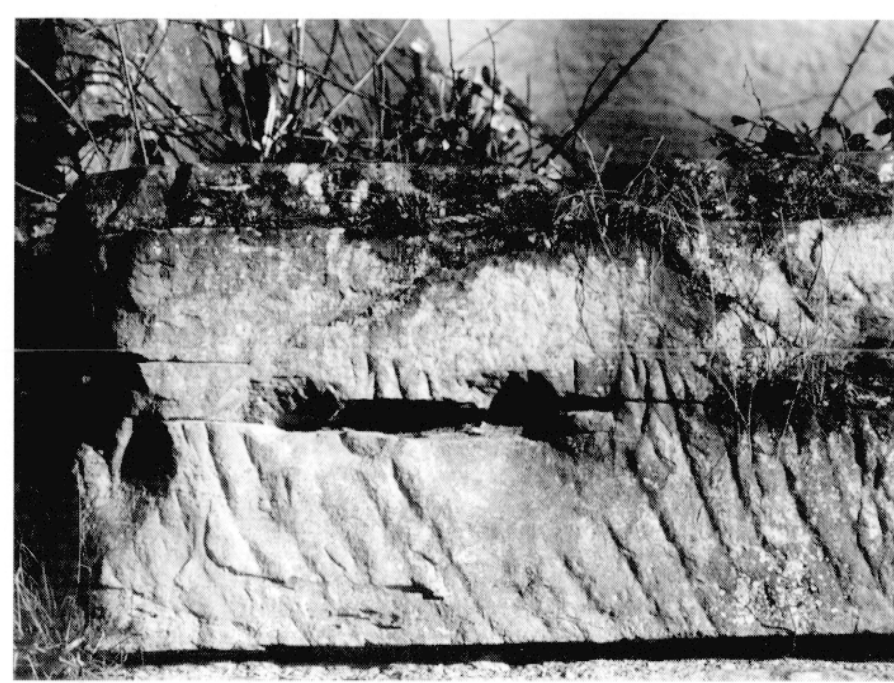

Fig. 128 - Limboîtures préparant l'extraction restée inachevée d'une couche de roche de faible épaisseur ( $35 \mathrm{~cm}$ environ) dans la partie centrale de la carrière du Kriemhildenstuhl, Bad Dürkheim (photo P. Adam).

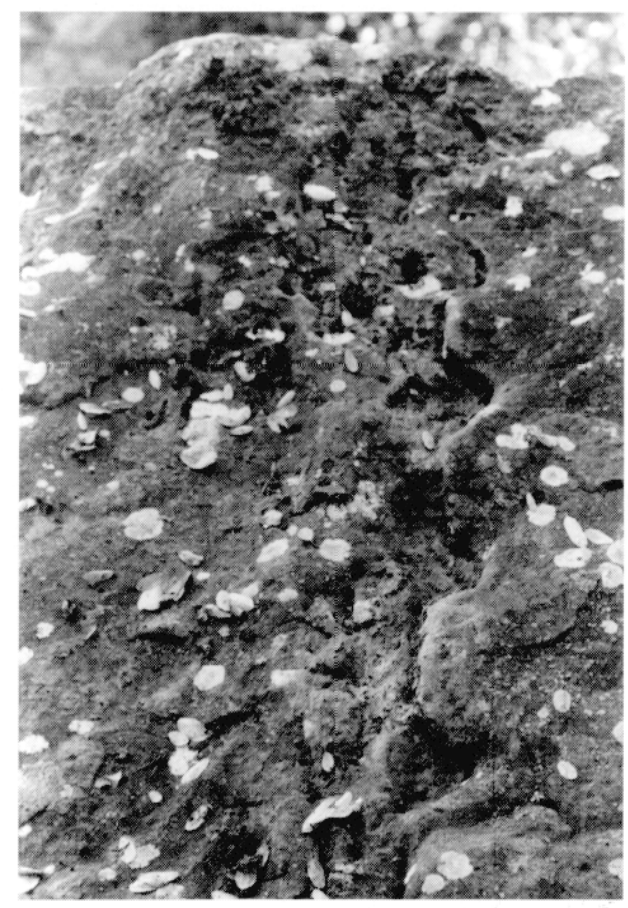

Fig. 129 - Alignement d'emboîtures dans la partie nord-est de la carrière du Kriemhildenstuhl, Bad Dürkheim (photo P. Adam).

opération qui nécessite un travail soigné, ce qui n'aurait sans doute guère été aisé avec un tel outil dans les sections étroites des emboîtures. Connu en Gaule méditerranéenne par ses empreintes (Bessac, 1996), un instrument du type mortaisoir ${ }^{*}$, un outil à percussion lancée muni de deux tranchants plats perpendiculaires à son manche, comparable aux exemplaires modernes, a pu être également utilisé dans cette région. Mais, une fois de plus, l'absence d'étude minutieuse des traces correspondantes nous empêche d'aller au-delà des suppositions concernant l'usage de cet outil dans le nord-est de la Gaule et en Rhénanie.

La disposition et l'espacement des logements de coin dépendent surtout des propriétés de la roche et de la capacité du carrier à évaluer le plus précisément possible la cohésion du substrat, contrairement à une hypothèse ancienne de J. Röder (1965) d'une évolution chronologique des emboîtures*. Une masse en fer servait à percuter les coins, une fois ceux-ci placés dans leurs logements ${ }^{101}$. De la Rhénanie, deux exemplaires nous sont parvenus, dont un du Kriemhildenstuhl (fig. 125, $\mathrm{n}^{\circ} 5$ ) et le second des galeries souterraines de la Pellenz (Röder, 1957, fig. 5.2).

\section{L'EXPLOITATION VOSGIENNE DE LA CROIX-GUILLAUME}

Une technique légèrement différente fut adoptée pour extraire des parallélépipèdes à La Croix-Guillaume, un site gallo-romain de hauteur dans les Vosges mosellanes, à une quinzaine de kilomètres au sud de Sarrebourg (Moselle). L'exploitation romaine du grès dans le secteur des Vosges n'a fait l'objet que de quelques mentions et les carrières de La Croix-Guillaume constituent un cas d'autant plus exceptionnel qu'elles ont pu profiter récemment d'investigations archéologiques exhaustives (cf. Heckenbenner et Meyer, supra, p. 145154). Occupant un plateau de grès d'environ $7000 \mathrm{~m}^{2}$, ce site comprend une petite carrière de pierre de taille et plusieurs points d'extraction de faible étendue, en activité entre le $\mathrm{I}^{\mathrm{er}}$ et le $\mathrm{III}^{\mathrm{c}} \mathrm{s}$. de notre ère. Le détachement des blocs se pratiquait principalement à l'aide de coins métalliques enfoncés dans des emboîtures*, elles-

101. Contrairement aux habitudes de l'extraction moderne traditionnelle (Noël, 1968 ; Prigent, 1985 ; Bessac, 1996 ; Bessac et al., 1997), l'emploi de paumelles de fer ou de bois pour caler les coins dans leur logement ne semble pas courant en Gaule romaine, bien qu'une absence de leurs traces ne soit pas obligatoirement un critère décisif prouvant que cet outil n'a pas été utilisé pour l'extraction, puisque leurs empreintes se conservent mal dans certains types de roches. Voir à ce sujet l'analyse critique de A. Dworakowska (1988). 
mêmes confectionnées dans des tranchées d'encoignures* situées aussi bien à la base du bloc que dans un plan vertical sur les faces arrière et latérales du bloc. L'existence de lithoclases verticales et de joints de stratification horizontaux a favorisé cette technique de détachement vertical, utilisant quasi exclusivement l'effet de coins métalliques, et évitant l'opération de havage ${ }^{*} 102$.

\section{L'EXPLOITATION SOUTERRAINE DANS LA PELLENZ ET DANS LA VALLÉE DE LA BROHL}

Dans certaines carrières souterraines de l'Eifel, les Romains ont pratiqué une technique tout à fait différente de celle que l'on a observée dans les carrières à ciel ouvert, où l'on extrayait autant que possible la pierre parallèlement au lit de la roche.

Implantées dans un affleurement de tuf trachytique sur les bords de la vallée de la Brohl et dans la région de la Pellenz (fig. 118), à quelques kilomètres du Rhin, ces carrières ont suscité un grand intérêt archéologique dans les années cinquante, suite aux activités d'extraction modernes en surface, qui avaient provoqué de profonds bouleversements du terrain et fait apparaittre des galeries antiques. Ces recherches sont étroitement liées au personnage de J. Röder, dont le travail pionnier en ce domaine de la recherche archéologique est reconnu. Son approche a gardé jusqu'à nos jours une certaine actualité, même si certains aspects demandent à être précisés. L'importance que ce chercheur a donnée au cadre géologique et pétrographique, qui est indispensable pour une bonne compréhension des activités dans une carrière, place ses recherches sur de solides fondations, étant donné les multiples interactions entre la roche et la technique d'extraction. Près de 40 ans plus tard, en 1997, de nouvelles fouilles archéologiques ont été reprises par le Landesamt für Denkmalpflege dans le cadre du projet de mise en valeur touristique "Vulkanpark Osteifel ». Elles ont permis de vérifier l'ancienneté et l'importance des vestiges d'extraction à Kretz (Schaaff, 2000). Si l'analyse du mobilier n'est pas encore terminée, les premiers résultats semblent confirmer l'essentiel des observations de J. Röder.

102. Cette technique fut appliquée dans divers contextes géologiques dans le monde romain, comme le gress, le calcaire ou bien le granite (Röder, 1965, 1974 ; Drouot, 1977 ; Bessac, 1987).

\section{PROBLÈMES DE RECHERCHE}

Bien que l'on parle sans réserve des vestiges romains de la Pellenz et de la vallée de la Brohl, il convient de préciser qu'il a été procédé à l'extraction du tuf à diverses époques jusqu'à nos jours, et qu'une partie des carrières ne date par conséquent que d'une époque plus récente. Les activités romaines se sont concentrées presque exclusivement sur le banc supérieur des deux couches de tufs exploitables, qu'on appelle communément Römertuff (tuf des Romains). La reprise de l'exploitation du tuf au Moyen Âge, en revanche, a concerné le banc de roche situé à un niveau inférieur, ce qui a évité une réouverture des galeries plus anciennes. Les structures antiques, épargnées des destructions jusqu'au $\mathrm{XIX}^{e}$ s., furent cependant en grande partie victimes de l'exploitation moderne de la pierre ponce, au $\mathrm{XX}^{\mathrm{c}} \mathrm{s}$. Aussi, ne faut-il pas s'étonner que les vestiges romains encore conservés soient peu nombreux par rapport à l'extension originelle des carrières, connue par des découvertes fortuites d'artéfacts romains.

Avant d'aborder quelques aspects techniques et le fonctionnement de cette extraction souterraine, il n'est pas inutile de rappeler un certain nombre des problèmes que posent les recherches en question, et dont la connaissance est primordiale pour une compréhension critique des vestiges d'extraction. J. Röder (1957, p. 222) lui-même désigne ses travaux comme " [...] erste umfassende Darstellung der antiken Tuffsteingewinnung dieser Gebiete [...] ", à savoir une première présentation globale de l'exploitation du tuf dans cette région, basée d'une part sur le recueil d'observations faites antérieurement par différents intervenants comme H. Lehner (1921), et d'autre part sur ses propres recherches sur le terrain.

L'équipe de J. Röder a rencontré de nombreuses difficultés sur le terrain, car l'examen de galeries souterraines est une opération dangereuse : risques d'effondrement d'un ciel de carrière*, de pénétration de l'eau dans les espaces vides, de glissement des déblais, etc. C'est en partie pour cela que la proportion explorée du site est relativement faible par rapport à l'étendue supposée des carrières antiques, puisqu'elle se réduit à une zone d'environ $60 \mathrm{~m} \times 60 \mathrm{~m}$. En outre, il est tout à fait regrettable que la documentation photographique, à laquelle le fouilleur a attaché une grande valeur, n'ait pas été publiée plus abondamment, voire intégralement, d'autant plus qu'elle semble extrêmement riche, et que 


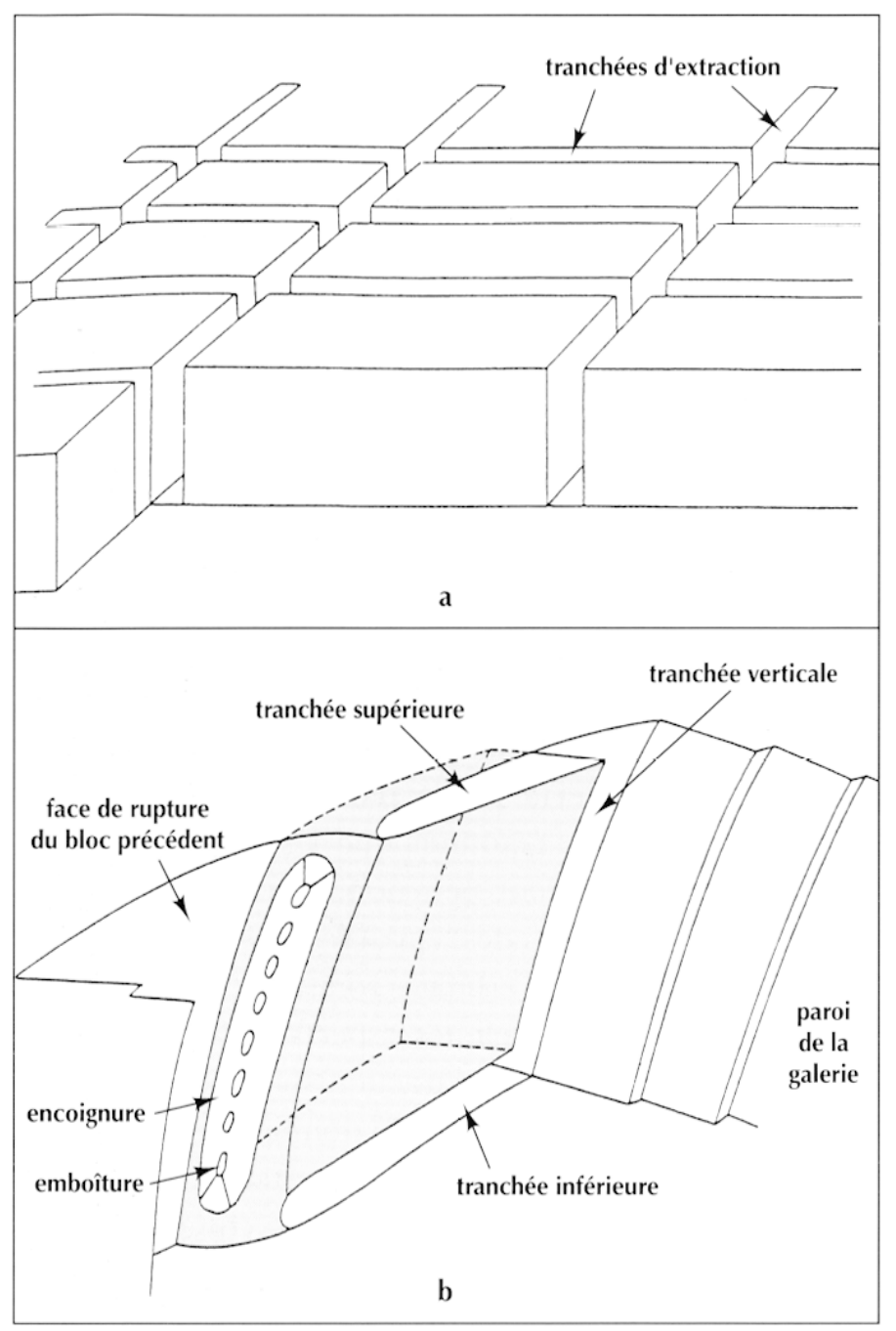

Fig. 130 - Schémas d'extraction dans une galerie souterraine : a, en lit; b, en délit (dessin modifié d'après Röder, 1957).

les fouilles récentes ont montré un état de conservation sensiblement plus médiocre des vestiges.

Il faut également rappeler les critères qui ont permis de dater ces carrières souterraines. L'origine antique de l'exploitation des gisements de tuf dans ces petites vallées perpendiculaires au Rhin est incontestablement attestée par des sources épigraphiques, dont nous avons déjà parlé. Toutefois, le mobilier archéologique (céramique, autels funéraires et votifs, sarcophages, etc.) trouvé anciennement dans les souterrains et aidant à cerner des phases d'activité ne provient dans la plupart des cas d'aucun contexte stratigraphique. Des inscriptions rupestres, signalées par H. Lehner (1921) ont été malheureusement victimes de l'exploitation moderne. Il faut souligner qu'une partie des vestiges d'extraction a pu être datée avec certitude lors des fouilles récentes avec la mise au jour d'un mobilier céramique en contexte stratigraphique (Schaaff, 2000, tabl. 19).

\section{DISPOSITION DES CARRIÈRES ET TECHNIQUE D'EXTRACTION}

Tandis que les galeries souterraines dans la vallée de la Brohl ont été aménagées en gradins et exploitées selon le schéma habituellement employé dans les carrières à ciel ouvert, soit une extraction en lit, parallèlement aux lits naturels de la roche ${ }^{103}$, une extraction perpendiculaire aux lignes de stratification a supplanté cette technique dans les galeries souterraines de la Pellenz, et en particulier dans la zone située entre les villages de Kruft et Plaidt (fig. 130). On procédait dans ce milieu souterrain à une extraction verticale, dite en délit ${ }^{*}$, telle que le plan de rupture du bloc était perpendiculaire aux plans de la stratification naturelle de la roche. La plus grande dimension du futur bloc, qui occupait toute la hauteur de la galerie, se trouvait dans un plan vertical. La roche se prêtait dans cette zone à un tel mode d'extraction du fait que son banc était presque dépourvu de fissures et de joints de stratification, qui auraient pu imposer des contraintes dimensionnelles. On parle aussi d'un tranchage vertical sur la hauteur du front. Le détachement des monolithes s'effectuait à partir de quatre tranchées creusées autour du bloc dont une, creusée en forme de " V ", constituait une encoignure", dans laquelle on insérait des coins d'extraction pour provoquer une rupture à l'arrière du bloc. Connue également des exploitations traditionnelles de tuffeau en Anjou et en Touraine au XXc $\mathrm{X}^{\mathrm{c}}$ s. (Prigent, 1985), cette technique était employée par les carriers romains en Rhénanie et s'est perpétuée dans la même région jusqu'au haut Moyen Âge, où elle était principalement liée à l'extraction de sarcophages. La question de son origine, que l'on peut vraisemblablement situer dans le monde grec, est toujours sujette à controverses.

L'épaisseur du banc de tuf exploité dans la Pellenz. variant entre $2 \mathrm{~m}$ et $3 \mathrm{~m}$, les galeries ne pouvaient atteindre qu'une hauteur maximale de $2 \mathrm{~m}$ à $2,5 \mathrm{~m}$, puisqu'il fallait laisser en place suffisamment de roche

103. Il fallait ouvrir des salles de vastes dimensions pour pouvoir appliquer cette technique dans des galeries souterraines. Pour les vestiges d'extraction dans la valléc de la Brohl, voir Röder, 1959a. 
saine pour garantir la stabilité des galeries (fig. 131). Les carriers cherchaient néanmoins à extraire un maximum de matériau de ce banc peu épais dans des galeries d'une largeur moyenne de 3,6 m (12 pieds romains), dont certaines ont été élargies au cours de la progression en salles de largeur double ou triple de celle de la galerie originelle. Les fronts de taille ${ }^{*}$ étaient partagés en deux pans correspondant à la largeur de deux parallélépipèdes, dont l'enlèvement a entraîné la progression de la galerie. Les blocs qui occupaient toute la hauteur de la galerie ont été extraits en alternance à droite et à gauche. Un nombre de blocs plus élevé était toutefois extrait lorsqu'on élargissait une galerie. Par contre, l'apparition de défauts naturels, sous forme d'inclusions de pierre ponce dans les parties supérieures du banc, obligeait les carriers à contourner certaines masses rocheuses. L'accès au réseau souterrain se faisait de plain-pied par le flanc de coteau ou par des puits de faible diamètre $(0,90 \mathrm{~m}$ à $1 \mathrm{~m}$ ), creusés à partir du niveau de la surface et débouchant sur les bords des galeries ou dans des niches creusées à cet effet dans leurs parois.

La conduite des opérations nous est connue grâce aux tranchées d'extraction abandonnées à divers stades de leur progression. Flles montrent que les carriers romains creusaient dans un premier temps une tranchéc horizontale, située au ras du plafond, ensuite une saignée verticale, correspondant à la hauteur du futur bloc, puis une troisième, en sens horizontal, au niveau du sol ${ }^{104}$. La largeur des tranchées était tracée au préalable sur le front de taille. L'opération de havage dans ces espaces souterrains était contrariée par un certain nombre de problèmes auxquels le carrier devait faire face lorsqu'il maniait l'outil, du fait de la disposition spécifique des tranchées et de l'exiguïté de l'espace de travail. De même, la forme des tranchées, dont la profondeur varie selon les dimensions des blocs entre $0,60 \mathrm{~m}$ et $0,80 \mathrm{~m}$, et dont la largeur à l'ouverture s'élève en moyenne à $20 \mathrm{~cm}$ alors qu'elle se rétrécit vers le fond jusqu'à 2 ou $3 \mathrm{~cm}$ (Röder, 1957, p. 257), est en grande partie imposée par les différentes positions de travail que le carrier devait prendre.

104. Il existait sans doute plusicurs variantes dans la conduite de ces općrations à l'époque romaine. On sait que les carricrs modernes des exploitations de tuffeau en Anjou pratiquaient le creusement des tranchées selon les ordres suivants : dessous-tranchée latérale-dessusencoignure, dessous-dessus-tranchée latérale-encoignure ou bien tranchée latérale-dessous-dessus-encoignure (Prigent, 1985).

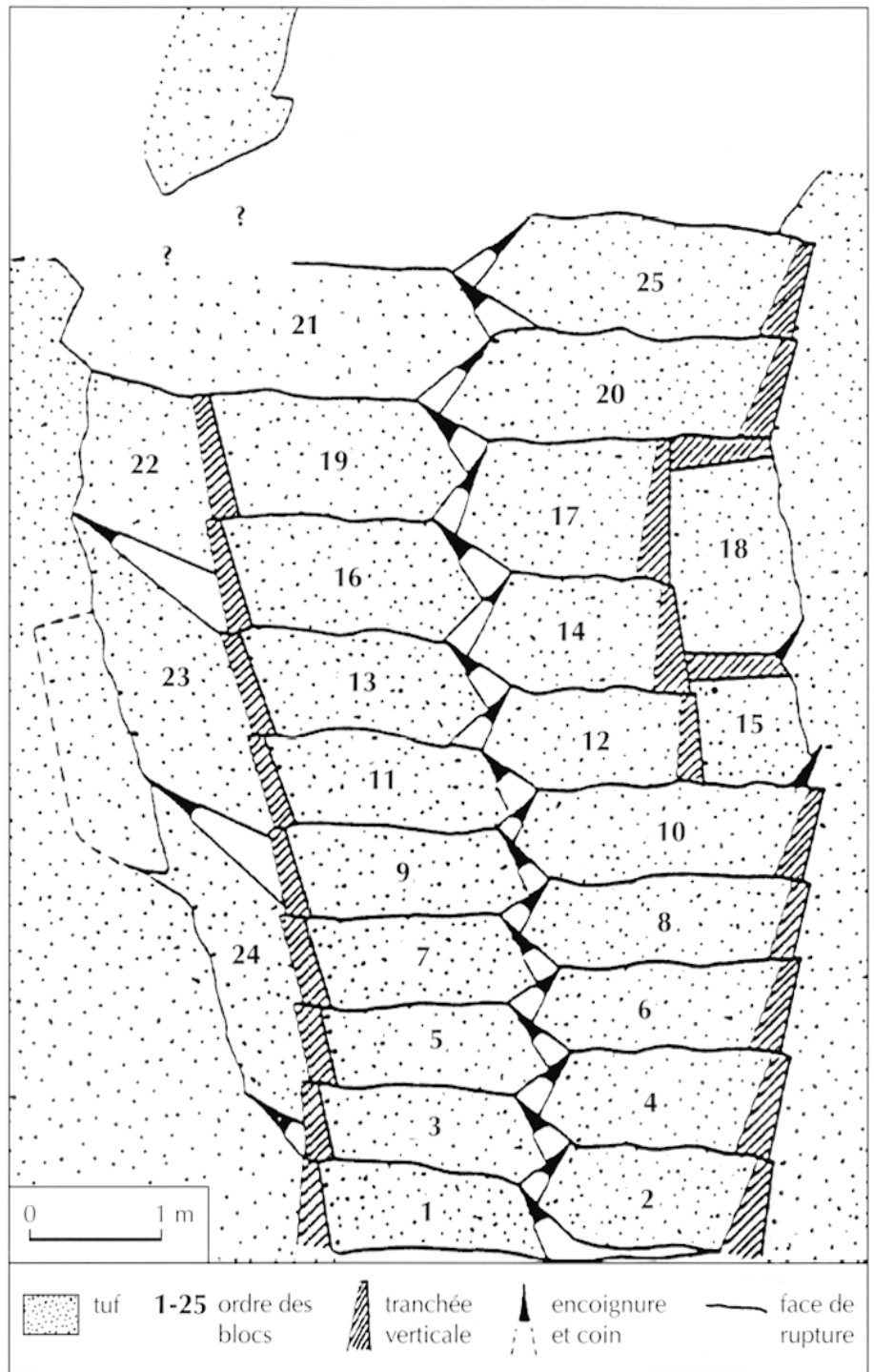

Fig. 131 - Schéma de principe de l'extraction dans une galerie souterraine de Kruft (Pellenz) : vue de dessus (dessin modifié d'après Röder; 1957).

Le creusement des saignées supérieures s'avérait particulièrement difficile, d'autant que leur position élevée exigeait un échafaudage ou la présence d'un bloc assurant cette fonction. Le carrier, alors debout face au bloc, devait soit guider son pic d'extraction selon un mouvement semi-circulaire dans un plan vertical pardessus sa tête, soit frapper en biais, afin d'atteindre la paroi à une hauteur de $2 \mathrm{~m}$ à $2,5 \mathrm{~m}$. L'inclinaison de l'outil, qui évitait la première position particulièrement pénible, permettait en outre de creuser plus précisément le fond de la tranchée, inaccessible à une frappe de face. Étant donné ces contraintes ergonomiques, il n'était guère possible d'obtenir une paroi supérieure plane, et 
celle-ci se présente, dans les exemples étudiés, légèrement incurvée vers le fond. Cette technique entrainait une réduction constante de la hauteur du front de taille, à laquelle il fallait s'opposer régulièrement par une rectification du ciel de carrière.

Des marques de carrier incisées sur certains fronts de taille au niveau d'irrégularités rocheuses peuvent également être mises en rapport avec l'opération de havage, voire la coordination de cette tache ${ }^{105}$. Ces marques rupestres figurent sur les parois d'une galerie souterraine à Krety, dans la Pellenz. (Röder, 1957, fig. 15 et 26.2). Leur emplacement en hauteur correspond aux limites inféricures des saignées de ciel des blocs correspondants et fournit ainsi une explication convaincante de leur fonction. Selon J. Röder, ces marques représenteraient en effet les directives d'un personnel d'encadrement chargé de coordonner les opérations d'extraction et de déterminer à l'avance l'emplacement des tranchées* en fonction de la qualité du substrat (Röder, 1957 , p. 261-263). Le fait que la roche comporte à ces endroits des hétérogénéités au niveau du ciel de carrière explique la démarche des carriers, obligés de modifier la position des saignées supérieures par rapport aux précédentes, et vient étayer l'hypothèse de J. Röder.

Pour creuser la tranchée verticale, proche de la paroi de la galerie, une petite tranchée étroite était tout d'abord réalisée, puis élargic latéralement et ensuite approfondie, ainsi que l'on procède pour l'abattage d'un arbre. Le creusement de cette tranchée verticale ne s'effectuait pas sur la totalité de la hauteur en une fois mais en plusieurs paliers, de sorte que le carrier terminait d'abord une première partie sur l'ensemble de la largeur, avant d'attaquer la roche restante. Dans ce contexte, les intervenants étaient confrontés à un sérieux problème de rétrécissement des tranchées en profondeur. Tandis que dans les carrières à ciel ouvert l'ouvrier pouvait changer de côté pour continuer le creusement en sens opposé, il était obligé, dans les galeries souterraines, d'élargir la tranchée pour tenir compte de son inévitable rétrécissement. Cet élargissement contribuait également à éviter un frottement violent des mains contre les parois rocheuses. Tandis que cet élargissement

105. Des marques rupestres de caractère fonctionnel sont commues dans de nombreuses carrières romaines de pierre de taille (Sprater, 1948 ; Röder, 1969 ; Monthel, Pinette, 1977 ; Dworakowska, 1983 ; Bessac, 1986b, 1988b, 1996). entraînait une perte de temps considérable, on laissait au contraire le rétrécissement se produire en fin de creusement lorsque celui-ci ne pouvait plus gêner le maniement de l'outil - un moyen de réduire le temps passé à l'opération de havage. Afin de diminuer les risques de blessure lors du maniement de l'outil d'extraction, les carriers ont également décalé les tranchées verticales par rapport à la paroi latérale de la galerie, de sorte que les parois de galerie présentent de légers ressauts (Röder, 1957, fig. 8, 23.3, 24 et 26.2).

Les saignées inféricures, creusées horizontalement au niveau du sol de carrière, n'ont pas pu être examinées à cause des déblais entassés dans les galeries. Même si nous ignorons leur apparence physique exacte, on peut imaginer que leur emplacement, à proximité immédiate du sol de la galerie, posait différents problèmes lors de. leur creusement : le carrier était contraint de s'accroupir sur le sol, en face du front de taille, et de guider l'outil d'extraction obliquement devant lui. La linéarité du fond de la tranchée, nécessaire pour garantir une bonne rupture du substrat, devait souffrir de cette posture qui rendait difficile un travail de précision.

Le bloc à extraire étant isolé sur trois de ses faces par des tranchées, il était nécessaire d'aménager pour les coins d'extraction une encoignure ${ }^{*}$ verticale, c'est-à-dire une saignée continue en forme de " $V$ », avant de procéder au détachement du bloc à partir de sa face arrière (fig. 130). De même que la tranchée latérale, l'encoignure était subdivisée pour le creusement en plusieurs petites saignées, chacune étant elle-même réalisée en plusieurs passes et, verticalement, en différents paliers. Elle était creusée sur toute la hauteur du bloc obliquement dans le front de taille*

Au fond de l'encoignure ${ }^{*}$, au niveau de la face arrière du bloc, le carrier confectionnait, sur une profondeur de $0,20 \mathrm{~m}$ et dans un espace extrêmement réduit, des emboîtures indépendantes dont le nombre, entre 7 et 9 , et l'espacement varient en fonction de la hauteur du bloc et des caractéristiques de la roche. Les coins étaient en fer comme en témoignent ceux qui ont été découverts dans les déblais d'une galerie romaine dans la Pellenz (fig. 132). Deux d'entre eux portent des inscriptions (Röder, 1957, p. 229, fig. 5.4 et 5.5). Ils étaient vraisemblablement placés sans l'intermédiaire de paumelles*, dont on n'a pas trouvé trace. Une fois les coins mis en place, le carrier les a percutés sur toute la hauteur du bloc à l'aide d'une masse dont un exemplaire nous est 
également parvenu (Röder, 1957, fig. 5.2), avant que le bloc détaché ne tombe dans la galerie, sans doute sur un lit de pierres préalablement dressé.

La rupture du bloc lors d'un tranchage vertical sur la hauteur du front résulte principalement d'une action combinée des forces de cisaillement, générées par le poids du bloc suspendu à la paroi, et de l'effet des coins métalliques. Ainsi, les fissures partant des emboîtures parcourent la roche pour rejoindre, selon le plus court chemin, le fond de la tranchée verticale opposée, à condition qu'aucune irrégularité dans le substrat ne provoque une déviation de leur progression. Les faces de rupture subsistantes montrent que l'arrachage des monolithes engendrait des surfaces de rupture plus ou moins irrégulières. Une surface de rupture concave pouvait entraîner le rejet du bloc, puisqu'elle cmpêchait d'obtenir un produit fini conforme aux exigences de la commande. Il est difficile d'évaluer quelle était la cause principale de ces mauvaises ruptures: des parois d'emboîture irrégulières, une percussion déséquilibréc ou l'existence de défauts naturels au sein du substrat? Un travail de rectification des fronts de taille" était de toute façon indispensable avant la reprise de l'extraction. Les mêmes opérations se répétaient dans la seconde moitić de la galerie pour l'extraction d'un bloc dont l'encoignure était contiguë à la précédente.

Enfin, lorsque la qualité de la roche justifiait une telle démarche, les carriers, au risque d'un effondrement du ciel, ont également extrait des parallélépipèdes dans le plafond des galeries, perpendiculairement au sens de la progression. Ils procédaient alors selon la méthode que nous venons de décrire, en aménageant trois saignées vers le haut, dans le ciel d'une galeric, avant de provoquer la rupture du bloc à partir d'une quatrième tranchée. Abstraction faite de la position de travail délicate, le danger auquel le carrier s'exposait à cette occasion était extrême, puisque le bloc risquait de tomber prématurément dans la galerie pendant l'opération de détachement - raison qui n'est probablement pas étrangère au fait que cette démarche soit restée exceptionnelle. Le souci d'une rentabilisation maximale du gisement au mépris des contraintes de sécurité s'exprime également dans l'affaiblissement des piliers de soutènement suite à l'extraction de blocs prismatiques. Plus fréquemment, les carriers ont enlevé des monolithes des parois latérales et provoqué ainsi un léger élargissement
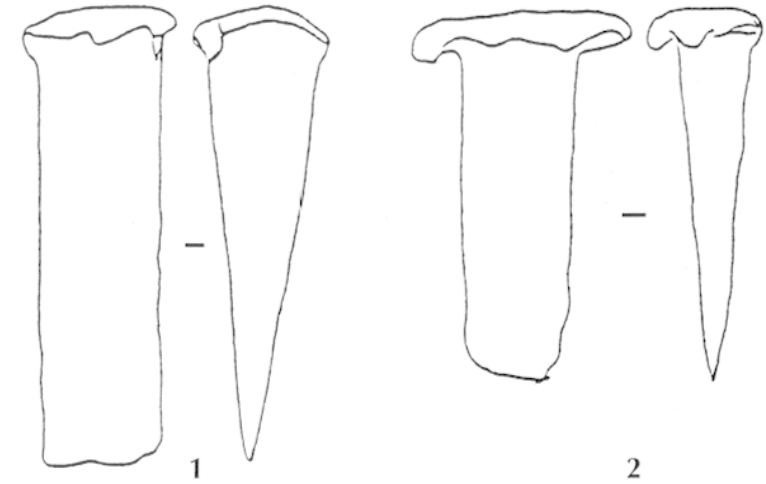

2

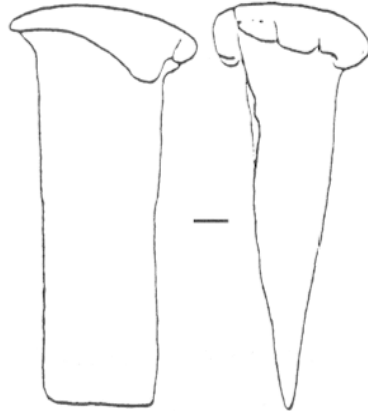

3

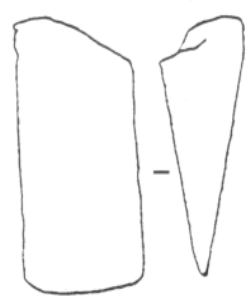

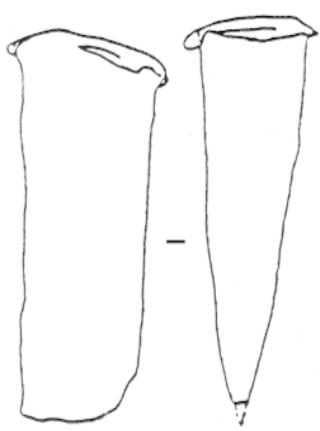

4

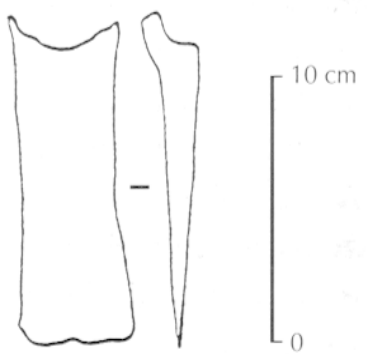

Fig. 132 - Coins d'extraction romains découverts dans la galerie Idylle de Kruft (Pellenz) (dessin modifié d'après Röder, 1957).

des galeries. Bien que le schéma d'extraction employé dans l'axe des galeries soit ici généralement appliqué, on observe quelques variations, comme la confection de deux encoignures verticales associées à deux saignées horizontales (Röder, 1957, fig. 9). Rien ne nous empêche toutefois de penser que ces vestiges appartiennent à unc phase d'extraction ultérieure pendant laquelle les carriers ont profité d'aménagements existants pour extraire quelques blocs des parois latérales (Schaaff, 2000, p. 27).

L'extraction entrânait forcément une accumulation importante de déchets au fur et à mesure de la progression, de l'équarrissage et du débitage des éléments bruts 
à proximité immédiate du front d'abatage ${ }^{* 106}$. Une gestion des déblais rigoureuse et parfaitement organisée était indispensable pour garantir un bon fonctionnement de la carrière et s'opposer en permanence au risque de son comblement. Ce problème était d'autant plus aigu dans les carrières souterraines de cette région que la largeur et la hauteur des galeries étaient réduites. Pour le résoudre et éviter de pénibles travaux d'évacuation jusqu'à la surface, les carriers ont adopté ici la solution la plus économique: en les gardant dans le réseau souterrain, ils ont entassé les déblais dans des galeries épuisées, sous forme de murs le long des parois des galeries, ou bien au-delà du puits le plus proche, afin de ne pas gêner la circulation dans l'exploitation (Schaaff, 2000, p. 26).

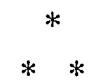

Autrefois lieux d'un travail éprouvant, sources d'information aujourd'hui, les carrières romaines que nous venons de décrire n'ont pas livré tous leurs secrets. Les données multiples amassées lors des études anciennes et des observations récentes ne révèlent

106. L'examen des déblais a permis d'identifier des déchets de taille résultant d'une première mise en forme des monolithes, dont la taille principale s'effectuait vraisemblablement dans des ateliers installés en surface (Röder, 1957, p. 250). qu'une faible part de la richesse scientifique dont pourraient témoigner ces documents archéologiques de premier ordre. Elles nous ont permis d'aborder l'organisation et les techniques, questions auxquelles une relecture des vestiges et l'investigation de nouveaux sites permettraient de répondre plus finement encore.

Mais d'autres sujets d'égale importance n'ont été qu'effleurés, sinon ignorés. Je pense principalement aux hommes qui ont laissé leur empreinte sur ces lieux, aux soldats qui nous ont signalé leur présence par leurs inscriptions, et dont le rôle et le statut dans ces carrières proches des frontières dynamiques du nord-est de la Gaule restent à élucider. Je pense aux propriétaires des multiples lieux d'extraction, et à tous les intervenants qui restent sans nom ni visage. L'étude des traces qu'ils ont laissées permettra aux archéologues de restituer l'image de ces communautés qui vécurent de la pierre.

\section{Nota bene}

Je voudrais témoigner une reconnaissance particulière à J.-C. Bessac pour ses conseils et son soutien constant. Mes remerciements vont également à $\mathrm{P}$. Adam pour ses encouragements et sa contribution à l'illustration de cet article, ainsi qu'à M. Vacca-Goutoulli, D. Heckenbenner, N. Meyer et F. Cognot qui ont bien voulu m'accueillir sur leurs chantiers de fouille. 